\title{
STABILITY OF THE COUETTE FLOW AT HIGH REYNOLDS NUMBERS IN TWO DIMENSIONS AND THREE DIMENSIONS
}

\author{
JACOB BEDROSSIAN, PIERRE GERMAIN, AND NADER MASMOUDI
}

Abstract. We review works on the asymptotic stability of the Couette flow. The majority of this paper is aimed toward a wide range of applied mathematicians, and there is an additional section aimed toward experts in the mathematical analysis of PDEs.

\section{Contents}

1. Hydrodynamic stability at high Reynolds number 374

2. Linear dynamics in dimension $d=2 \quad 382$

3. Nonlinear dynamics in dimension $d=2 \quad 386$

4. Linear dynamics in dimension $d=3 \quad 389$

5. Nonlinear dynamics in dimension three 392

6. Statement of the theorems 395

7. Rigorous mathematical proofs

8. Related questions 404

9. Open problems 405

About the authors $\quad \lcm{408}$

References

Here we provide a review on the stability of the two-dimensional (2D) and threedimensional (3D) Couette flow in the Navier-Stokes equations at high Reynolds number, focusing mainly on the recent sequence of works [11 14, 17, 18. Our goal is to provide a general discussion of the wider physical context for these works and those which are related and, specifically, to discuss how they fit into the wider field of hydrodynamic stability. To this purpose, we have attempted to make $\S ₫ 16$ accessible to a wide range of applied mathematicians and mathematical analysts, whereas 97 is targeted toward experts in the analysis of partial differential equations (PDEs).

Received by the editors March 6, 2018.

2010 Mathematics Subject Classification. Primary 76-02, 35-02, 76E05, 76E30, 35B25, 35B35, $35 \mathrm{~B} 34$.

Key words and phrases. Hydrodynamic stability, high Reynolds number, Couette flow, subcritical transition.

The first author was supported by NSF DMS-1552826.

The second author was supported by NSF DMS-1501019.

The third author was supported by DMS-1716466. 


\section{Hydrodynamic stability at high Reynolds number}

1.1. The Navier-Stokes and Euler equations. We start by recalling the incompressible Navier-Stokes equations in dimension $d=2$ or 3 , with inverse Reynolds number $\nu:=\mathbf{R e}^{-1} \geq 0$

$$
\left\{\begin{array}{l}
\partial_{t} v+v \cdot \nabla v=-\nabla p+\nu \Delta v \\
\nabla \cdot v=0 \\
v(t=0)=v_{\text {in }}
\end{array}\right.
$$

where the velocity $v=\left(\begin{array}{l}v^{1} \\ v^{2}\end{array}\right)$ or $v=\left(\begin{array}{l}v^{1} \\ v^{2} \\ v^{3}\end{array}\right)$ takes values in $\mathbb{R}^{d}$, the pressure $p$ is a scalar, and $v \cdot \nabla$ stands for $\sum_{i=1}^{d} v^{i} \partial_{i}$. If the Reynolds number is infinity (hence $\nu=0$ ), then the system is known as incompressible Euler equations, which reads

$$
\left\{\begin{array}{l}
\partial_{t} v+v \cdot \nabla v=-\nabla p \\
\nabla \cdot v=0 \\
v(t=0)=v_{\text {in }}
\end{array}\right.
$$

Both $v$ and $p$ are functions of the time and space variables $(t, x, y)$ or $(t, x, y, z)$, in space dimensions 2 or 3 , respectively. Boundary conditions and external forces can also be added to these equations and are necessary for most nontrivial hydrodynamic stability questions.

This article is concerned with hydrodynamic stability at high Reynolds number (i.e., in the singular limit $\nu \rightarrow 0$ ). See the texts [41,107, 126] for reviews on the wider theory of hydrodynamic stability; see especially [126] for an extensive review of the literature and a detailed account of the development of the field. Here we will only present a brief introduction especially targeting mathematicians. The basic problem is to consider a given equilibrium $11 u_{E}$ for either (NS) or (E), and to study the dynamics of solutions which are close to $u_{E}$ in a suitable sense (we will be more precise later). Especially, to answer the simple question of whether or not the flow remains close to $u_{E}$ in certain norms or not, and if so, which norms. Hence, if we write the solution $v=u_{E}+u$, the exact evolution equations for the perturbation become

$$
\left\{\begin{array}{l}
\partial_{t} u+u_{E} \cdot \nabla u+u \cdot \nabla u_{E}+u \cdot \nabla u=-\nabla p+\nu \Delta u \\
\nabla \cdot u=0 \\
u(t=0)=u_{\text {in }}
\end{array}\right.
$$

where $u_{\text {in }}$ is the initial perturbation. For hydrodynamic stability questions, naturally $u_{\text {in }}$ is assumed initially small in certain norms (in the limit $\nu \rightarrow 0$, the question can be sensitive to this choice, as we will see). The vast majority of work is focused on laminar equilibria - simple equilibria in which the fluid is moving in well-ordered layers (as opposed to equilibria with chaotic streamlines). Typical examples include shear flows in various geometries such as pipes and channels, vortices or vortex columns, and flows in concentric cylinders [41, 107, 126]. However, even for these simple configurations, surprisingly little is understood about the near-equilibrium dynamics in the limit $\nu \rightarrow 0$, especially at the mathematically rigorous level. When studying this limit, it is natural to first consider $\nu=0$, which,

\footnotetext{
${ }^{1}$ As remarked above, in order to get nontrivial equilibria, we usually need to impose boundary conditions or an external force field. However, let us ignore this for the moment, as it is not directly relevant to our discussions.
} 
as a general rule, will be strictly easier than studying the singular limit. Indeed, significantly more is known about stability theory for the Euler equations $(\nu=0)$ than for the Navier-Stokes equations $\nu>0$ in the limit $\nu \rightarrow 0$.

1.2. Linear theory and its limitations. It is often implicitly presumed that linear stability is equivalent to nonlinear stability in practical settings for physically relevant systems. However, even the very early linear studies of Rayleigh 99. and Kelvin [70] seemed in contradiction with experimental observations, particularly the famous experiments of Reynolds [102]. Indeed, in [102, Reynolds pumped fluid through a pipe under various conditions and demonstrated that at sufficiently high Reynolds number, the laminar equilibrium becomes spontaneously unstable. Despite the observed nonlinear instability, to this day, there is no evidence of any linear instabilities in 3D pipe flow for any finite Reynolds number [41,126]. Perhaps even more troubling is that even for laminar flows for which the linearization has unstable eigenvalues at high Reynolds numbers, experiments and computer simulations normally display instabilities which are different than those predicted by the linear theory (and at lower Reynolds numbers) [1,28, 107, 126]. These phenomena are known in fluid mechanics as subcritical transition or bypass transition, and are completely ubiquitous in 3D hydrodynamics.

To understand the subtleties of subcritical transition, it is important to first be more precise about the meaning of "linear stability" and "nonlinear stability". The linearization of (1.1) is obtained simply by dropping the quadratic nonlinearity in (1.1):

$$
\left\{\begin{array}{l}
\partial_{t} u+u_{E} \cdot \nabla u+u \cdot \nabla u_{E}=-\nabla p+\nu \Delta u \\
\nabla \cdot u=0, \\
u(t=0)=u_{\text {in }} .
\end{array}\right.
$$

Write (1.2) abstractly as

$$
\left\{\begin{array}{l}
\partial_{t} u=\mathcal{L}_{E} u \\
u(t=0)=u_{\text {in }}
\end{array}\right.
$$

The simplest notion of linear stability is spectral stability.

Definition 1.1 (Spectral stability). Given a Hilbert space $H$, let $\sigma\left(\mathcal{L}_{E}\right)$ be the spectrum of $\mathcal{L}_{E}$. The equilibrium $u_{E}$ is called spectrally stable if $\sigma\left(\mathcal{L}_{E}\right) \cap$ $\{c \in \mathbb{C}: \operatorname{Re} c>0\}=\emptyset$. The equilibrium is called spectrally unstable if $\sigma\left(\mathcal{L}_{E}\right) \cap$ $\{c \in \mathbb{C}: \operatorname{Re} c>0\} \neq \emptyset$ and is called neutrally stable if it is spectrally stable but $\sigma\left(\mathcal{L}_{E}\right) \cap\{c \in \mathbb{C}: \operatorname{Re} c=0\} \neq \emptyset$.

Many classical theories of hydrodynamic stability are focused on studies of spectral stability (see [126] and the references therein). In the context of shear flows at high Reynolds number, the most famous results are Rayleigh's inflection point theorem [41,99], and its refinement due to Fjørtoft [49] regarding the stability of shear flows in the 2D Euler equations $(\nu=0)$. These results (unfortunately) extend to planar 3D shear flows via Squire's theorem [40,41,110]. Squire's theorem for inviscid flows states the following. If the 3D Euler equations linearized around the shear flow $u_{E}=(f(y), 0,0)$ have unstable eigenvalues, then so do the 2D Euler equations linearized around $u_{E}=(f(y), 0)$ (the converse implication being obvious). Therefore, for any shear flow of this type, spectral stability in 2D implies spectral stability 
in 3D. This is unfortunate because it gives the false impression that most of the interesting aspects of hydrodynamic stability can be found in the $2 \mathrm{D}$ equations. As we will see, hydrodynamic stability in $2 \mathrm{D}$ and $3 \mathrm{D}$, at high Reynolds numbers, is quite distinct.

Spectral stability is not the only relevant definition of linear stability. In particular, we have the following distinct definition, which was suggested as more natural in fluid mechanics by Kelvin [70, Orr [95, and later Case [29] and Dikiur [39].

Definition 1.2 (Lyapunov linear stability). Given two norms $X$ and $Y$ (often assumed the same), the equilibrium $u_{E}$ is called linearly stable (from $X$ to $Y$ ) if

$$
\|u\|_{Y} \lesssim\left\|u_{\mathrm{in}}\right\|_{X}
$$

where $u$ solves (1.2).

Even for finite-dimensional systems of ordinary differential equations (ODEs), Definitions 1.1 and 1.2 are not equivalent for neutrally stable, nondiagonalizable linear operators (the infinite-dimensional analogue being nonnormal operators). The canonical example of this phenomenon, which is directly relevant for hydrodynamic stability as we will see, is given by the linear system

$$
\partial_{t}\left(\begin{array}{l}
x_{1} \\
x_{2}
\end{array}\right)=\left(\begin{array}{cc}
0 & -1 \\
0 & 0
\end{array}\right)\left(\begin{array}{l}
x_{1} \\
x_{2}
\end{array}\right) .
$$

The matrix $\left(\begin{array}{cc}0 & -1 \\ 0 & 0\end{array}\right)$ is not diagonalizable. It has spectrum reduced to $\{0\}$, and is therefore neutrally stable, but it is not Lyapunov stable: solutions are given by

$$
\left\{\begin{array}{l}
x_{1}(t)=x_{1}(0)-t x_{2}(0) \\
x_{2}(t)=x_{2}(0)
\end{array}\right.
$$

and therefore grow. The above example can be modified to make it spectrally stable and Lyapunov linearly stable:

$$
\partial_{t}\left(\begin{array}{l}
x_{1} \\
x_{2}
\end{array}\right)=\left(\begin{array}{cc}
-\nu & -1 \\
0 & -\nu
\end{array}\right)\left(\begin{array}{l}
x_{1} \\
x_{2}
\end{array}\right) .
$$

As pointed out by Orr in 1907 95, nonnormality implies that a large transient growth is possible. As $\nu \rightarrow 0$, the system degenerates, leading to the optimal estimate

$$
\sup _{t}\left\|\left(x_{1}(t), x_{2}(t)\right)\right\| \lesssim \nu^{-1}\left\|\left(x_{1}(0), x_{2}(0)\right)\right\|
$$

Hence, while the problem is linearly stable for all $\nu>0$, the solutions are (in general) growing very large as $\nu \rightarrow 0$. As Orr pointed out in 95, we could expect that any prediction of nonlinear stability from a linear system of this type should be problematic as $\nu \rightarrow 0$, a fact which has been greatly expanded on in modern times (see below). Many problems of hydrodynamic stability display transient behavior reminiscent of (1.4) in the limit $\nu \rightarrow 0$. See below and [98, 107, 113, 114] and the references therein for more details. 
1.3. Quantitative nonlinear stability and transition thresholds. The limitations of linear theory being increasingly clear, we must turn to more nonlinear stability theories in order to connect with physical observations. The simplest notion of nonlinear stability is that of Lyapunov.

Definition 1.3 (Lyapunov (nonlinear) stability). Given two norms $X$ and $Y$ (often assumed the same), the equilibrium $u_{E}$ is called stable (from $X$ to $Y$ ) if, for all $\epsilon>0$, there exists a $\delta>0$ such that if

$$
\left\|u_{\text {in }}\right\|_{X}<\delta \quad \Longrightarrow \quad\|u(t)\|_{Y}<\epsilon \text { for all } t>0
$$

We say $u_{E}$ is unstable if it is not stable.

There are many works in fluid mechanics and related fields dedicated to proving spectral instability (for the linearized problem) and to proving that spectral instability implies nonlinear instability; see, e.g., [50,52, 61, 62, 108, to name a few in the mathematics community. This theory has led to the clearer understanding of many physical phenomena in hydrodynamics [41, 65, 80, 100] but it does not help us to understand the phenomenon of subcritical transition as it does not help us explain instabilities which are not directly associated with a spectral instability.

Probably the most flexible and powerful general theory for nonlinear stability results in hydrodynamics is that of Arnold for the 2D Euler equations, introduced in [3], now usually called the Energy-Casimir method. This theory provides nonlinear stability in the sense of Definition 1.4 for $X=Y=H^{1}$ for certain equilibria, such as shear flows satisfying certain convexity hypotheses and vortices [42,89, 120]. Variations have been extended to a great many other settings, for example magnetohydrodynamics and kinetic theory; see the review article [66] and the more recent works in stellar mechanics 63, 64 for more information. The fundamental idea behind this technique is to use the large number of extra conservation laws available to the 2D Euler equations, the so-called Casimirs, to find a conserved quantity which is locally convex, coercive in $H^{1}$, and for which the equilibrium is the local minimizer. There are several limitations of the theory: it is mostly restricted to two-dimensional settings and infinite Reynolds number (recall that, in the presence of boundaries, one can find examples of $2 \mathrm{D}$ shear flows in a channel which are stable at infinite Reynolds numbers by Arnold's theory, but which are spectrally and nonlinearly unstable in $L^{2}$ at arbitrarily high (but finite) Reynolds numbers [50,62]).

The above discussion suggests that we are still lacking a good mathematical understanding of subcritical transition (recall that, for equilibria which are linearly stable, subcritical transition refers to the spontaneous transition to a turbulent state often observed at high Reynolds numbers in experiments and computer simulations). It has also been observed, even by Reynolds [102], that at which Reynolds number and precisely how it occurred seems unusually sensitive to the details of the experimental setup. In [70, Kelvin suggested the idea that maybe such equilibria are nonlinearly stable for all finite Reynolds numbers in the sense of Definition 1.3 , but that the system becomes increasingly sensitive to perturbations as the Reynolds number increased. This suggests that what we should study is a quantification of $\delta$ in Definition 1.3 . 
Definition 1.4 (Quantitative asymptotic stability). Given two norms $X$ and $Y$, the equilibrium $u_{E}$ is called asymptotically stable (from $X$ to $Y$ ) with exponent $\gamma$ if

$$
\left\|u_{\text {in }}\right\|_{X} \ll \nu^{\gamma} \Longrightarrow \begin{cases}\|u(t)\|_{Y} \ll 1 & \text { for all } t>0 \\ \|u(t)\|_{Y} \rightarrow 0 & \text { as } t \rightarrow \infty\end{cases}
$$

We say $u_{E}$ is unstable if it is not stable.

We remark that the polynomial dependence $\nu^{\gamma}$, as well as the necessity of two norms $\|\cdot\|_{X}$ and $\|\cdot\|_{Y}$, is dictated in part by empirical observation. As we will see, the choice of final norms $\|\cdot\|_{Y}$ is severely constrained by linear dynamics, however, the initial norms $\|\cdot\|_{X}$ are not. In fact, there is no unique, natural physical choice of $X$. Moreover, numerical experiments [101] and weakly nonlinear heuristics suggest that $\gamma$ should depend nontrivially on the choice of norms $X$ and $Y$. While this has not yet been proved for the Navier-Stokes equations, it has been proven that in the 2D Euler equations, the nonlinear dynamics is sensitive to the regularity. Specifically, in [37] it was recently shown that the earlier work on the Euler equations [14] cannot be extended to any Gevrey classes not already covered in the original work. A closely related result had also already been established for the Landau damping of the Vlasov-Poisson equations in a periodic box [9]; see $\$ 8.2$ below for more discussion on how Landau damping is related to hydrodynamic stability questions.

We also remark that this definition should be adapted in situations where there is a continuum of equilibria, and the solution might relax, as $t \rightarrow \infty$, to an equilibrium different from the one which is initially perturbed (as is for instance the case for the 2D Euler equation [14, which will be briefly discussed below).

In case an equilibrium is asymptotically stable, the above definition raises the following question (given $X$ and $Y$ ):

\section{What is the smallest exponent $\gamma>0$ for which $u_{E}$ is quantitatively asymptotic stable?}

As suggested above, the motivation for Definition (1.4) and Question (Q1) go all the way back to Kelvin; however, modern authors have expanded on these concepts further (see, e.g., 101,107,114 and the references therein). Another very important question, which is a little more vague, is to understand precisely how the instability occurs near the stability threshold.

$$
\begin{aligned}
& \text { If } \gamma \text { is the optimal exponent in Q1), } \\
& \text { for a } \gamma^{\prime}<\gamma \text {, classify all solutions satisfying } \\
& \qquad \nu^{\gamma} \lesssim\left\|u_{\text {in }}\right\|_{X} \lesssim \nu^{\gamma^{\prime}} \text { and } \sup _{t \geq 0}\|u(t)\|_{Y} \gtrsim 1 .
\end{aligned}
$$

When considering only the question of spectral stability or instability of planar shear flows, Squire's theorem suggests that one can focus exclusively on the 2D case (see, e.g., 41]). However, one gets very different answers in $2 \mathrm{D}$ and $3 \mathrm{D}$ when considering the nonlinear questions Q1 and Q22. Indeed, it is often observed that subcritical instabilities appear at lower Reynolds numbers than eigenvalue instabilities, and that even when 2D eigenvalue instabilities are present, fully 3D, nonmodal instabilities are often still observed to be dominant [107,126. Similarly, 
we see major differences when comparing our 2D and 3D works on planar Couette flow for sufficiently smooth data (see below and [14,17] for the 2D works and [1, 12 . for 3D). One cannot escape the reality that any study of 2D hydrodynamic stability at high Reynolds number is of very limited physical relevance to 3D flows.

\subsection{Inviscid damping and asymptotic stability at infinite Reynolds num-}

ber. While question (Q1) explicitly concerns only $\nu>0$, it is natural to ask, if $\gamma=0$, whether some kind of stability holds for the inviscid problem $\nu=0$. For nontrivial shear flows, one can verify that this is not possible unless $d=2$ (see $\$ 4$ for more discussion); indeed, it is reasonable to imagine that every nontrivial, laminar 3D equilibrium is nonlinearly unstable in the 3D Euler equations (regardless of how strong one is willing to choose the initial norm $X$ ). However, for $d=2$, it turns out in some cases to be possible not only to have Lyapunov stability, but also to identify the long-term dynamics and have a kind of asymptotic stability even if $\nu=0$. In [14], it was demonstrated that near the shear flow $u_{E}=(y, 0)$ (with $(x, y) \in \mathbb{T} \times \mathbb{R})$, for $u_{i n}$ sufficiently small and sufficiently smooth, there exists some $u_{\infty}$ such that

$$
u(t, x, y) \rightarrow\left(\begin{array}{c}
u_{\infty}(y) \\
0
\end{array}\right) \quad \text { as } t \rightarrow \infty
$$

strongly in $L^{2}$ and weakly in $H^{1}$ (and analogously also $\lim _{t \rightarrow-\infty} u(t)=\left(u_{-\infty}(y), 0\right)$ for some $\left.u_{-\infty}\right)$. Notice that this is much stronger than the kinds of stability results one derives via the Energy-Casimir method. See also the recent work [69] which proves similar results for a bounded channel in $y$.

This convergence back to equilibrium can seem surprising, since it does not follow from one of the classical relaxation mechanisms - energy dissipation or decay through dispersion of waves. This particular mechanism is known as inviscid damping and is a close relative of Landau damping in plasma physics. It was proved that Landau damping provides a similar stability for the Vlasov-Poisson equations in $\mathbb{T}^{d}$ in Mouhot and Villani's breakthrough work 93 ; (see $\$ 8.2$ for more discussion on Landau damping). Inviscid damping was observed in the linearized Euler equations first by Orr in 1907 [95]; see 92 for more discussion on this effect. Landau damping is considered fundamental to understanding the dynamics of collisionless and weakly collisional plasmas by the physics community [26, 105] and it has been speculated that inviscid damping should play a related role in understanding the dynamics of the 2D Euler equations [6, 56, 91, 106, 128, with applications to cyclotron dynamics [31] and atmospheric sciences [90, 109, 121]. See [21, 81, 82, 123, 125, 127, 133, 135] for mathematical works on inviscid damping in the linearized 2D Euler equations.

1.5. The Couette flow. It turns out that the simplest equilibrium to study is the plane periodic Couette flow:

$$
u_{E}(x, y)=\left(\begin{array}{l}
y \\
0
\end{array}\right) \quad(\text { if } d=2), \quad u_{E}(x, y, z)=\left(\begin{array}{l}
y \\
0 \\
0
\end{array}\right) \quad(\text { if } d=3) .
$$

Here "plane" differentiates this flow from the Taylor-Couette flow, the analogous equilibrium between concentric, rotating cylinders [41, while "periodic" refers to the choice $x \in \mathbb{T}$ (the circle, which we identify with $[0,2 \pi]$ with periodic boundary 
conditions). Specifically, we consider the following geometry for the domain:

- In dimension $d=2,(x, y) \in \mathbb{T} \times \mathbb{R}$.

- In dimension $d=3,(x, y, z) \in \mathbb{T} \times \mathbb{R} \times \mathbb{T}$.

From now on we will simply refer to this flow as the Couette flow.

Why study the Couette flow in this domain specifically? There are several good reasons:

- The Couette flow is a canonical problem and has played the role of a benchmark for different approaches to understanding hydrodynamic stability for well over a century (see [41, 107, 126] and the references therein). This is due in part to its simplicity and to the fact that it is a solution of the Navier-Stokes equations for all $\nu \geq 0$.

- The domain of infinite extent in the $y$ direction avoids the presence of walls, which can add very subtle complications to hydrodynamic stability problems (see also 99.2 for more discussion).

- The shear gives rise to a mixing phenomenon (sometimes referred to as "filamentation") which has profound implications for the dynamics and is mostly a stabilizing effect; however, this effect is very weak at low wave numbers in $x$. The periodic domain in the $x$ direction removes such problematic wave numbers. The periodicity is most similar to the mixing which occurs around a radially symmetric vortex; however, studying the stability of vortices at high Reynolds number is significantly more difficult (see [21, 54, 77]).

- The reduction to periodic boundary conditions in $x$ and infinite extent in $y$ is common in the physics literature both for formal asymptotic analysis [32, 107,114 and (at least the periodicity in $x$ ) for numerical studies.

1.6. Summary of the known stability results on the plane periodic Couette flow. Many works have studied the stability of the plane periodic Couette flow and its variants in attempt to get answers to Questions (Q1) and (Q2). For experiments on Couette flow, see [24, 35, 112], and for computational studies, see, e.g., 45, 96, 101. In [114, the authors suggested a weakly nonlinear toy model which couples transient growth in the linear problem to a caricature of the nonlinearity in an attempt to capture some of the aspects of subcritical transition. For more work along this general line, see, e.g., 4, 5, 32, 55, 84, 119. As far as rigorous analysis goes, there is the work of Romanov [104] who first proved nonlinear stability at all Reynolds number for the Couette flow in an infinite pipe geometry. Later, the works [73, 79] estimate $\gamma$ rigorously assuming numerically obtained resolvent estimates. See [126] for further references on Couette flow as well as references focusing on related flows, such as the plane Poiseuille flow and the cylindrical pipe flow.

The series of works [11,14, 17, 18, gives values of $\gamma$ for which the answer to (Q1) is positive, but it also gives a very fine description of the perturbed flow, and how it relaxes to zero. Results depend on the topology which is considered 2 either

\footnotetext{
${ }^{2}$ Roughly speaking, a finite Gevrey $\mathcal{G}^{\lambda ; s}$ norm requires an average decay slightly stronger than $e^{-\lambda|\xi|^{s}}$ in frequency, while a finite Sobolev norm requires an average decay slightly stronger than $|\xi|^{-s}$.
} 
TABLE 1

\begin{tabular}{|c|c|c|}
\hline & $X$ & References \\
\hline \multirow{2}{*}{ dimension $d=2$} & Sobolev & {$[18$} \\
\cline { 2 - 3 } & Gevrey & {$[14,17]$} \\
\hline \multirow{2}{*}{ dimension $d=3$} & Sobolev & {$[13,122]$} \\
\cline { 2 - 3 } & Gevrey & {$[11,12]$} \\
\hline
\end{tabular}

Sobolev norms $H^{s}$ or Gevrey norms $\mathcal{G}^{\lambda ; s}$ :

$$
\begin{aligned}
\|f\|_{H^{s}} & =\left(\sum \int|\hat{f}(\xi)|^{2}(1+|\xi|)^{2 s} d \xi\right)^{1 / 2}, \\
\|f\|_{\mathcal{G}^{\lambda ; s}} & =\left(\sum \int|\hat{f}(\xi)|^{2} e^{2 \lambda|\xi|^{s}} d \xi\right)^{1 / 2} .
\end{aligned}
$$

Due to the fast mixing of the Couette flow, one cannot hope to obtain uniform-in- $\nu$ stability with $Y$ in higher $H^{s}$ spaces besides $H^{1}$ in two dimensions (due to the vorticity transport equation); in $3 \mathrm{D}$, one cannot expect uniform-in- $\nu$ stability in any $H^{s}$ with $s>0$. However, if one rewinds the dynamics by the mixing of the shear flow $y+\frac{1}{2 \pi} \int_{0}^{2 \pi} u^{1}(\tau, \alpha, y, z) d \alpha$, one can hope to obtain uniform stability in arbitrarily high norms. Below we refer to the profile

$$
f(t, x, y)=u\left(t, x+t y+\int_{0}^{t} \frac{1}{2 \pi} \int_{0}^{2 \pi} u^{1}(\tau, \alpha, y, z) d \alpha d \tau, y, z\right) .
$$

The terminology is from dispersive equations, where the idea of rewinding by the leading order oscillatory linear behavior is classical. Control of the profile in high regularity precisely characterizes the dynamics, for example, localizing the solution in frequency.

The obtained results are summarized in Table 1.

We want to draw attention to the very recent work of Wei and Zhang [122, which proved asymptotic stability in velocity variables and enhanced dissipation at $H^{2}$ regularity with $\gamma \leq 1$. These results were not expected (at first they seem inconsistent with numerical simulations [101]) and were quite impressive. In [13], optimal $H^{s}$ estimates on the profile were obtained for $\gamma \leq 3 / 2$, and it is possible that the methods of Wei and Zhang might extend to obtain optimal $H^{s}$ estimates for $\gamma \leq 1$. In two dimensions, the $\gamma$ is known to depend on regularity: the results of 37. show that the Gevrey results of [14, 17] cannot be extended to Sobolev spaces (though the $\gamma \leq 1 / 2$ estimate in [18] might not be sharp). However, it is possible that in 3D, Sobolev vs Gevrey changes only the method of transition; e.g., $\gamma=1$ in both Sobolev and Gevrey, but in Gevrey only streaks are possible slightly above the threshold as proved in 12 but in Sobolev perhaps other, more nonlinear, instabilities are also possible (such alternative instabilities are indeed observed in experiments 101]).

Physicists were in agreement that (for 3D) $\gamma \geq 1$, and the only question they considered is whether $\gamma=1$ or $\gamma>1$; each has been conjectured (see 5.1 and [32, 101, 107, 114]). However, strictly speaking, a full mathematical proof that $\gamma \geq 1$ is not currently carried out to our knowledge. Computer simulations of "smooth" initial perturbations have often returned values slightly over-estimating the (suspected) answer of $\gamma=1$ for Gevrey spaces (see [45,101] and the references therein). 
Indeed, the general trend has been a steady decrease down to 1 , which was only reported relatively recently in [44. However, $\gamma=31 / 20$ was reported for "rough" initial perturbations in [101; this exponent seems more robust to computationand matches closely the value $\frac{3}{2}$ appearing in 13 - but interestingly, does not match [122. We are not currently aware of any 2D simulations of this type.

From a mathematically rigorous perspective, we have significantly less information regarding the classification of instabilities (Q2). In general, this problem appears to be significantly harder than (Q1). The only (rigorous) results in this direction are that of [12, which consider the Gevrey norm in 3D with initial data $\nu \lesssim\left\|u_{i n}\right\|_{\mathcal{G}^{s, \lambda}} \lesssim \nu^{2 / 3+\delta}$ for $\delta>0$ small. In this work, we prove that only one instability is possible. The instability is driven by a distinctly $3 \mathrm{D}$, linear, nonnormal transient growth known as the lift-up effect [46, 74]; see \$4 below for more discussion. A related work is that of [82, in which the authors prove that inviscid damping matching the linearized problem is false in general for the nonlinear 2D Euler equations with perturbations of Couette flow arbitrarily small in $H^{s}$ for $s<3 / 2$.

Remark 1.5. It is inaccurate to suggest that all physical settings should be compared to Gevrey or Sobolev (or $L^{2}$ ) norms. One should instead measure the size of the perturbations in the various norms after restricting to scales larger than a suitable dissipative length-scale. Essentially, below this scale, viscosity dominates and the difference between the norms is no longer relevant.

1.7. Notations and Fourier analysis conventions. Given two quantities $A$ and $B$, the notation

$$
A \lesssim B
$$

means that there exists a constant $C$ such that $A \leq C B$, while

$$
A \ll B
$$

means that $A \leq c B$ for a constant $c$ which is sufficiently small (depending on the context).

We will use the Fourier transform frequently in 2D and 3D. In 2D we use

$$
\widehat{f}(k, \eta)=\frac{1}{2 \pi} \int_{\mathbb{T} \times \mathbb{R}} e^{i(k X+\eta Y)} f(X, Y, Z) d X d Y,
$$

and in $3 \mathrm{D}$ we use

$$
\widehat{f}(k, \eta, \ell)=\frac{1}{(2 \pi)^{3 / 2}} \int_{\mathbb{T} \times \mathbb{R} \times \mathbb{T}} e^{i(k X+\eta Y+\ell Z)} f(X, Y, Z) d X d Y d Z .
$$

Note that $k, \ell \in \mathbb{Z}$ whereas $\eta \in \mathbb{R}$. It will be very important in the following to distinguish between zero and nonzero frequencies in $x$ and $X(k=0$ or $k \neq 0)$. We will denote the orthogonal projection onto the modes $f_{0}$ and $f_{\neq}$, respectively,

$$
f_{0}=\frac{1}{2 \pi} \int_{\mathbb{T}} f d X \quad \text { and } \quad f_{\neq}=f-f_{0} .
$$

\section{LINEAR DYNAMICS IN DIMENSION $d=2$}

In two dimensions, it is most natural to consider the problem in the vorticity formulation. Recall that the vorticity $\omega$ of the perturbation is given by

$$
\omega=\partial_{x} u^{2}-\partial_{y} u^{1} \text {. }
$$


We will also use the stream function $\phi$, which satisfies

$$
\Delta \phi=\omega, \quad u=\nabla^{\perp} \phi .
$$

The equation in vorticity form reads

$$
\left\{\begin{array}{l}
\partial_{t} \omega+y \partial_{x} \omega+\nabla^{\perp} \phi \cdot \nabla \omega=\nu \Delta \omega \\
\Delta \phi=\omega .
\end{array}\right.
$$

2.1. Coordinate transform. For small data and small $\nu$, we expect the transport by the Couette flow in (2.2) to be dominant. Hence, it is natural to switch to coordinates in which this transport is canceled or "modded out". To that effect, set

$$
\left\{\begin{array}{l}
X=x-t y \\
Y=y
\end{array}\right.
$$

We will follow the convention that dependent variables which are capitalized are taken in the new coordinates $(X, Y)$, whereas lowercase is reserved for the original coordinates $(x, y)$. Hence, for instance

$$
\begin{aligned}
& U(t, X, Y)=u(t, x, y), \\
& \Omega(t, X, Y)=\omega(t, x, y) .
\end{aligned}
$$

Under this change of coordinates, differential operators transform as

$$
\begin{aligned}
& \left\{\begin{array}{l}
\nabla u=\nabla_{L} U, \\
\Delta u=\Delta_{L} U,
\end{array}\right. \\
& \quad \text { where } \nabla_{L}=\left(\begin{array}{c}
\partial_{X} \\
\partial_{Y}-t \partial_{X}
\end{array}\right) \text { and } \Delta_{L}=\nabla_{L} \cdot \nabla_{L}=\partial_{X}^{2}+\left(\partial_{Y}-t \partial_{X}\right)^{2} .
\end{aligned}
$$

In the new coordinates (2.3), the equation satisfied by $\Omega$ reads

$$
\left\{\begin{array}{l}
\partial_{t} \Omega+\nabla^{\perp} \Phi \cdot \nabla \Omega=\nu \Delta_{L} \Omega \\
\Delta_{L} \Phi=\Omega
\end{array}\right.
$$

Note that there was a crucial cancellation: the terms involving $\pm t \partial_{X} \Phi \partial_{X} \Omega$ cancel. Such a fortuitous cancellation will not hold in 3D. Linearizing this for small perturbations gives the very simple

$$
\partial_{t} \Omega=\nu \Delta_{L} \Omega .
$$

2.2. Enhanced dissipation. The fact that the dissipation of a passive scalar can be enhanced via advection by an incompressible velocity field is a well-known effect, going sometimes by the names "shear-diffuse mechanism", "relaxation enhancement", and "enhanced dissipation". It has been studied previously in linear problems in both the mathematics literature [7, 8, 19, 20, 33, 68, 77, 118, 137. (see also [53]) and in the physics literature [22, 43, 76, 85, 103. We remark that Lundgren 85. considered possible implications for the behavior of vortex filaments in turbulent flow, whereas Dubrulle and Nazarenko [43. suggested the possibility that the enhanced dissipation effect could have an important impact on Questions (Q1) and Q2 . This effect can be explained as follows: the advection transfers enstrophy to very high frequencies where it is then more rapidly dissipated. It was Kelvin [70] who first made the observation when he solved (2.6) via Fourier analysis in 1887.

First, the zero frequencies in $X$ are governed by the simple heat equation (recall that $\left.\Omega_{0}=\int \Omega d X\right)$,

$$
\partial_{t} \Omega_{0}=\nu \partial_{Y Y} \Omega_{0}
$$


Note that $\Omega_{0}(t, y)$ defines the vorticity of a shear flow by the Biot-Savart law in (2.6). The dissipation time-scale is $\sim \nu^{-1}$ and there is obviously no enhancement for these modes.

Next, we consider the nonzero frequencies in $X$. Applying the Fourier transform to (2.6) gives

$$
\partial_{t} \widehat{\Omega}(k, \eta)=-\nu\left[k^{2}+(\eta-k t)^{2}\right] \widehat{\Omega}(k, \eta) .
$$

If $k \neq 0$, then integrating the above ODE gives

$$
\begin{aligned}
\widehat{\Omega}(k, \eta, \ell) & =\exp \left(-\nu \int_{0}^{t}\left(k^{2}+(\eta-k s)^{2}\right) d s\right) \widehat{\Omega_{i n}}(k, \eta) \\
& \Longrightarrow|\widehat{\Omega}(k, \eta, \ell)| \lesssim \exp \left(-c \nu k^{2} t^{3}\right)\left|\widehat{\Omega_{i n}}(k, \eta)\right|
\end{aligned}
$$

for a fixed, small constant $c>0(1 / 12$ suffices $)$. Using that $x \in \mathbb{T}$, this implies that, in any reasonable norm (by the Biot-Savart law $U=\nabla_{L}^{\frac{1}{}} \Delta_{L}^{-1} \Omega$ ),

$$
\left\|\Omega_{k}(t)\right\|+\left\|U_{k}(t)\right\| \lesssim e^{-c \nu k^{2} t^{3}}\left\|\Omega_{\text {in }}\right\| .
$$

The exponent $\nu k^{2} t^{3}$ gives a dissipation time-scale $\sim \nu^{-1 / 3}$. This is very short compared to the dissipation time-scale $\nu^{-1}$ which is observed for zero frequencies in $X$. In more general linear advection-diffusion problems or linearized Navier-Stokes equations, precise, quantitative estimates on this enhanced dissipation effect are significantly harder to obtain; see, e.g., [8, 19, 33, 68, 77]. Notice that the effect becomes weak at low wave numbers; see $\$ 9.2$ for more discussion.

The mixing effect that gives rise to enhanced dissipation is a very common phenomenon in high Reynolds number fluid mechanics. One can see it when stirring milk into coffee as shown in Figure 1.
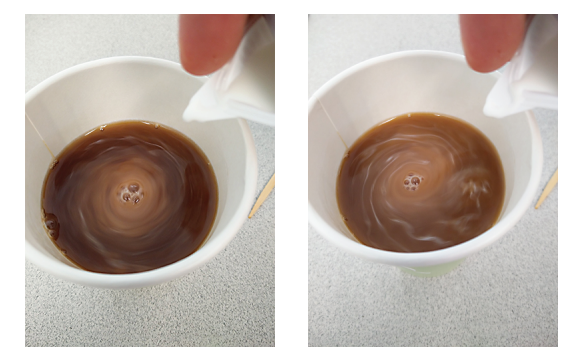

Figure 1. Milk mixing into an approximately axisymmetric vortex

2.3. Inviscid damping and the Orr mechanism. The enhanced dissipation already implies that the Couette flow should have a stabilizing effect. However, there is an additional, more subtle, inviscid effect. This was discovered by Orr in 1907 [95] and is often known now as inviscid damping, in part due to its connection with Landau damping in the kinetic theory of plasmas (see 8.2 . From the BiotSavart law, we have

$$
\widehat{U}(t, k, \eta)=-i\left(\begin{array}{c}
k t-\eta \\
k
\end{array}\right) \frac{1}{k^{2}+(\eta-k t)^{2}} \widehat{\Omega}(t, k, \eta) .
$$


We saw above that the vorticity experiences enhanced dissipation; since the full formula is a bit long, we will be content with the approximation $\widehat{\Omega_{\neq}}(t, k, \eta) \sim$

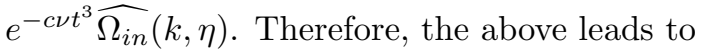

$$
\text { for } k \neq 0, \quad \widehat{U}(t, k, \eta) \sim-i\left(\begin{array}{c}
k t-\eta \\
k
\end{array}\right) \frac{1}{k^{2}+(\eta-k t)^{2}} \widehat{\Omega_{i n}}(k, \eta) e^{-c \nu k^{2} t^{3}} .
$$

This formula captures two important effects observed by Orr - one stabilizing and one destabilizing - together known as the Orr mechanism [95]:

(a) As $t \rightarrow \infty$, it is clear from the above formula that $\widehat{U}_{\neq}$, hence $U_{\neq}$, converges to 0 uniformly in $\nu$ (see below for quantitative statements).

(b) On the other hand, the denominator is minimal for $t=\frac{\eta}{k}$, which corresponds to a transient amplification if $\nu t^{3} \ll 1$.

Let us first discuss the damping (a). One can be more precise about the rate of decay and obtain the following estimates pointwise-in-time:

$$
\begin{aligned}
\left\|U_{\neq}^{1}(t)\right\|_{H^{s}} & \lesssim \frac{1}{\langle t\rangle}\left\|\Omega_{i n}\right\|_{H^{s+1}}, \\
\left\|U_{\neq}^{2}(t)\right\|_{H^{s}} & \lesssim \frac{1}{\langle t\rangle^{2}}\left\|\Omega_{i n}\right\|_{H^{s+2}} .
\end{aligned}
$$

Notice crucially that this damping is independent of Reynolds number, and indeed, clearly holds also if $\nu=0$; it would, however, depend on the size of the torus, were it not fixed. By undoing the coordinate change, the above gives decay for $u_{\neq}$in $L^{2}$, or in spaces with sufficiently small Sobolev exponent. After undoing the coordinate transform (2.3), this implies similar decay in $L^{2}$, however, higher $H^{s}$ norms in general experience slower decay (for $s<1$ ) or no decay at all $(s \geq 1)$. Like enhanced dissipation, the inviscid damping is weak at low wave numbers in $x$. In 2D fluid mechanics, the inviscid damping can be easily interpreted as being due to the vorticity being mixed rapidly by the Couette flow, which sends enstrophy to high frequencies. Since the Biot-Savart law damps high frequencies, these become less relevant to the velocity field and, eventually, only the shear flow remains. The mixing of the vorticity is often called "filamentation", and we remark that it results in a kind of inverse cascade of energy as the energy in the $x$-dependent modes disappears (in a finite energy, nonlinear problem, this energy would necessarily move into the $x$-independent modes; an analogous effect happens in Landau damping which causes plasmas to heat up as they undergo Landau damping).

In regard to the transient amplification alluded to in (b) above, observe that before the onset of enhanced dissipation, the vorticity $\Omega$ is essentially constant, so that

$$
\text { if } k \neq 0 \text { and } \nu t^{3} \ll 1, \quad \frac{\widehat{U}\left(t=\frac{\eta}{k}, k, \eta\right)}{\widehat{U_{i n}}(k, \eta)} \sim \frac{|\eta|+|k|}{|k|} .
$$

Therefore, if $|\eta| \gg|k|$, the velocity field is amplified by a large factor between $t=0$ and the critical time $t=\frac{\eta}{k}$. In physical terms, this transient growth is due to the fact that the mode of the vorticity in question is initially well mixed and then proceeds to unmix under the Couette flow evolution. See Figure 2 for how this mixing/un-mixing effect appears on each Fourier mode of the vorticity. The relevance of the Orr mechanism to hydrodynamic stability has been debated over the years; see, e.g., [95], [25], 83], and [126] for a detailed account of how 

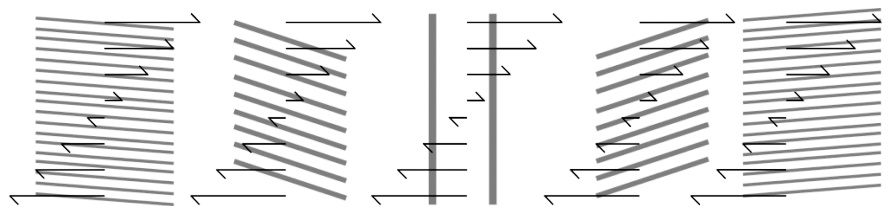

Figure 2. A visualization of the Orr mechanism: the stripes represent the level sets of a function on the torus, which is advected by the Couette flow, represented by the arrows (in analytical terms, represented are the level sets of the function $e^{i k(x-t y)+i \eta y}$ with $\eta / k \gg 1)$. The five pictures represent different moments of the evolution, with time increasing from left to right. One can see how the Couette flow first unwraps the field before wrapping it up again on the torus.

the literature on the topic developed. The importance of the Orr mechanism for nonlinear problems is discussed further in 3.2

\section{NONLINEAR DYNAMiCS IN DIMENSION $d=2$}

3.1. Shear flows as metastable states. As we just saw on the linear level, nonzero frequencies in $x(k \neq 0)$ of the velocity field are suppressed (in $L^{2}$ topology) via:

(a) inviscid damping, with a polynomial decay on a time scale $\sim 1$ (with respect to $\nu)$

(b) enhanced dissipation, with an exponential decay on a time scale $\sim \nu^{-1 / 3}$.

Both effects imply that, at the linear level, the dependence of $u$ on $x$ is erased in $L^{2}$ as time goes by; by the divergence-free condition, this implies that $u$ approaches a shear flow rapidly in $L^{2}$. Convergence of $u$ to a shear flow in higher norms follows after the enhanced dissipation time-scale $\nu^{-1 / 3}$. However, damping of modes $k=0$ is felt on the much longer time scale $\sim \frac{1}{\nu}$ (which ensures at least the ultimate convergence of the perturbation $u$ to 0 ). As a conclusion, on a linear level, shear flows are metastable states, or intermediate attractors, for the velocity field in $L^{2}$ on the time range

$$
1 \ll t \ll \nu^{-1} .
$$

More specifically, for $u_{M S}(y)=\left(u_{0}^{1}(y), 0\right)$, it holds that

$$
\left\|u(t)-u_{M S}\right\|_{L^{2}} \lesssim \frac{\epsilon}{\langle t\rangle} \quad \text { for } 1 \ll t \ll \nu^{-1} .
$$

Moreover, we have the following estimate for all $s>0$ for some $c>0$,

$$
\left\|u(t)-u_{M S}\right\|_{H^{s}} \lesssim \epsilon\langle t\rangle^{s-1} e^{-c \nu t^{3}} \quad \text { for } 1 \ll t \ll \nu^{-1} .
$$

Both of these estimates are sharp for the linear problem and involve implicit constants which are independent on $\nu$. Notice that for large $s$ there is a large transient growth before the decay ultimately dominates. This is a result of the transfer of enstrophy from low to high frequencies due to the mixing.

What about the nonlinear problem? Shear flows solve the 1D heat equation for $\nu>0$ and are stationary solutions for $\nu=0$, but are they metastable states? A positive answer to this question is one of the main results in [17, 18, (and in some sense [14]). One of the most important differences between the linear and nonlinear 
dynamics is the fact that the perturbation itself causes a small adjustment to the background shear flow. Specifically, the zero frequency of the first velocity component $u_{0}^{1}(t, y)$ is unaffected by the enhanced dissipation or inviscid damping and hence decays only on the very long $O\left(\nu^{-1}\right)$ time-scale. It follows that over long time-scales, this adjustment to the shear flow can have a very large effect on the solution. As a result, (2.3) is no longer necessarily the natural coordinate transformation (this problem is vaguely analogous to a quasi-linear scattering problem in dispersive equations, especially for the 2D Euler equations [14). As discussed further below, this is one of several major difficulties in the proofs of [14,17]. The situation in $3 \mathrm{D}$ is even more complicated.

3.2. Nonlinear resonances. The frequency cascade scenario. Now we arrive at the most fundamental difficulty in understanding the nonlinear dynamics near shear flows at high Reynolds number, specifically, the nonlinear resonances associated with the nonnormal transient growth in the linear problem. These are not "true" resonances as they are not associated with the spectrum of the linear problem, but rather, with the pseudo-spectrum (see, e.g., [113,114]). We will not dwell on such abstract questions, but rather take a direct, hands-on approach to understanding them. These nonlinear interactions are much simpler and much better understood in the 2D case - in 2D there is essentially one leading order nonlinear resonance, whereas the $3 \mathrm{D}$ case is far more complicated, and it is not clear precisely which interactions will dominate in which situations.

We shall use the coordinate transform conventions defined in $₫ 2$. As we saw in the previous section, the $(k, \eta)$ mode of $\Omega$ (the vorticity in the new variables) has a large effect on $\Phi$ (which is the stream function $\phi$, defined in (2.1), expressed in the new variables) at the critical time $t=\eta / k$, as can be seen from

$$
\widehat{\Phi}(t, k, \eta)=-\frac{\widehat{\Omega}(t, k, \eta)}{k^{2}+|\eta-k t|^{2}}
$$

(which is simply the Biot-Savart law). Physically, if $k \eta>0$, then the mode in the vorticity undergoes a transient unmixing at the critical time $\eta / k$ before ultimately mixing. One then intuitively separates the modes into those for which $\eta / k<t$ and those for which $\eta / k>t$ : the unmixing and mixing modes 3 Then, one imagines a three-wave nonlinear interaction which continues to transfer information from mixing to unmixing modes to sustain a nontrivial velocity field for long times. This effect was first demonstrated in 1968 in collisionless plasmas by the famous plasma echo experiments [88. Similar hydrodynamic echoes were observed in the $2 \mathrm{D}$ Euler equations near a radially symmetric vortex via experiments on a pure electron plasma in a strong magnetic field [130]. Figure 3 appeared originally in 88.

An echo is a nonlinear oscillation in which the velocity field (or electric field in the case of plasmas) decays after an initial disturbance but spontaneously becomes large again later as high-frequency information generated by nonlinear interactions unmixes. Notice that this is essentially a strong interaction between the linear transient growth coming from the Orr mechanism and the nonlinearity. Specifically,

\footnotetext{
${ }^{3}$ In plasma physics these are sometimes referred to as "phase mixing" and "antiphase mixing" modes.
} 


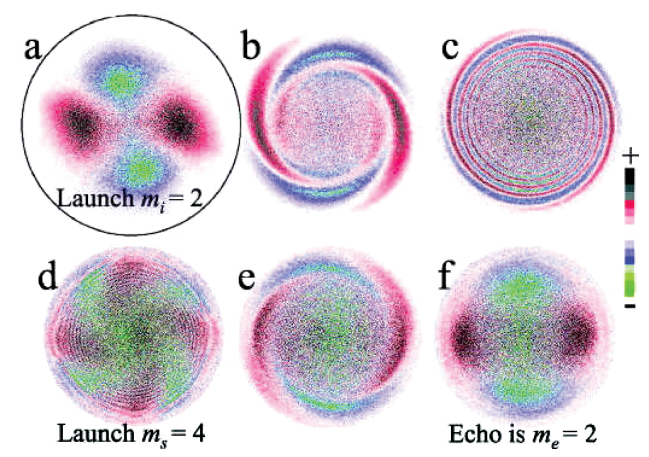

FIgURE 3. An experiment showing a hydrodynamic echo in the 2D Euler equations near a radially symmetric vortex. In other words, the geometry is circular rather than toroidal, and a vortex replaces the Couette flow, but the echo phenomenon remains valid. At the initial time (a), the Fourier mode 2 (in the angular variable) is excited by an exterior force, before Landau damping erases it in (b) and (c). At time (d), a new exterior force is applied to excite the Fourier mode 4, and it is subsequently erased by Landau damping in (e). But a nonlinear echo phenomenon (interaction between the modes 2 and 4 ) is responsible for the observation, at the later time (f), of a strong signal at the Fourier mode 2. Reproduced with permission of AIP Publishing from Physics of Plasmas 12, 055701, (2005); https://doi.org/10.1063/1.1885006 ([130, Figure $5])$.

the scenario we are most concerned with is the following cascade:

- Around the critical time $t=\frac{\eta}{k}$, the mode $\widehat{\Phi}(k, \eta)$ is linearly amplified.

- Through a nonlinear interaction, it causes the mode $\Omega(k-1, \eta)$ to grow...

- ... which, in turn, linearly amplifies $\widehat{\Phi}(k-1, \eta)$ around time $t=\frac{\eta}{k-1}$.

- And so on!

In order to understand and estimate more quantitatively the growth which can result from this scenario in the $2 \mathrm{D}$ Euler equations, we will next develop a toy model. Closely related studies have been done previously for the 2D Euler equations [116,117, and in some ways our toy model is simpler but also better suited to getting mathematically rigorous estimates on the dynamics.

The toy model. The echoes involve the interaction of a mode which is well mixed and a mode which is near the critical time. In terms of $\Omega$, the vorticity in the shifted coordinates, the well-mixed mode will be at a low wave number and the critical mode will be at a high wave number (since we are interested in long times). Hence, let us formally consider solutions to (2.5) of the form $\Omega=\Omega_{L}+\Omega_{H}$, where $\Omega_{L}$ consists only of low frequencies and $\Omega_{H}$ is a much higher frequency perturbation (but has much less enstrophy). Let us assume here that neither have contributions to the zero mode in $X$; let us further think of the low frequencies as a background 
that we are not so interested in, and we then linearize the equation on high frequencies to obtain

$$
\begin{aligned}
\partial_{t} \Omega_{H}+\nabla^{\perp} \Phi_{H} \cdot \nabla \Omega_{L}+\nabla^{\perp} \Phi_{L} \cdot \nabla \Omega_{H} & =\nu \Delta_{L} \Omega_{H}, \\
\Delta_{L} \Phi_{H} & =\Omega_{H} .
\end{aligned}
$$

We argue now that most terms can be dropped in the above: First, we set $\nu=0$, since the frequency cascade behavior which preoccupies us would typically occur before dissipation kicks in. Second, since $\nabla^{\perp} \Phi_{L}$ is decaying rapidly and longterm dynamics are of interest, we drop the term $\nabla^{\perp} \Phi_{L} \cdot \nabla \Omega_{H}$. Finally, since $\widehat{\Phi}_{H}(t, k, \eta)=-\frac{\widehat{\Omega}_{H}(t, k, \eta)}{k^{2}+|\eta-k t|^{2}}$, we see that the term involving $\partial_{Y} \Phi_{H}$ is the most problematic in $\nabla^{\perp} \Phi_{H} \cdot \nabla \Omega_{L}$, as near the critical time $t \sim \eta / k, \widehat{\partial} Y_{\Phi_{H}} \sim-k t \widehat{\Omega}_{H}$. Hence, we reduce to the (linear in $\Omega_{H}$ ) model equation

$$
\begin{aligned}
\partial_{t} \Omega_{H}-\partial_{Y} \Phi_{H} \partial_{X} \Omega_{L} & =0, \\
\Delta_{L} \Phi_{H} & =\Omega_{H} .
\end{aligned}
$$

Taking the Fourier transform of (3.1), the model becomes

$$
\partial_{t} \widehat{\Omega}_{H}(t, k, \eta)=-\sum_{k^{\prime} \neq 0} \int \frac{\eta^{\prime}\left(k-k^{\prime}\right)}{\left(k^{\prime}\right)^{2}+\left(\eta^{\prime}-k^{\prime} t\right)^{2}} \widehat{\Omega}_{H}\left(t, k^{\prime}, \eta^{\prime}\right) \widehat{\Omega}_{L}\left(k-k^{\prime}, \eta-\eta^{\prime}\right) d \eta^{\prime} .
$$

As $\Omega_{L}$ is concentrated at low frequencies, let us make the simplifying approximation that $\eta \approx \eta^{\prime}$ and $k^{\prime}=k \pm 1$. This gives us the model

$$
\partial_{t} \widehat{\Omega}_{H}(t, k, \eta)=-\sum_{k^{\prime}=k \pm 1} \frac{\eta\left(k-k^{\prime}\right)}{\left(k^{\prime}\right)^{2}+\left(\eta-k^{\prime} t\right)^{2}} \widehat{\Omega}_{H}\left(t, k^{\prime}, \eta\right) \Omega_{L}\left(k-k^{\prime}, 0\right) .
$$

The idea is that this model might be useful in estimating the leading order, longtime dynamics of how enstrophy in the high frequency perturbation $\Omega_{H}$ is transferred between modes in $X$ as a result of the interactions with the low frequencies. For a general solution, the simplifying assumptions we made in deriving (3.2) are all inaccurate, and we will not be able to say that (3.2) is necessarily an accurate model for the dynamics. Nevertheless, in [37] it was shown that at least in certain regimes, the toy model provides an accurate picture of the leading order nonlinear behavior. The echo construction for the Vlasov-Poisson equations [9] similarly shows that the analogous toy model there can be an accurate approximation; see 8.2. However, in [14,17, we instead use (3.2) to estimate the worst case scenario, that is, we use (3.2) to get an upper bound on how much enstrophy can be transferred from low frequencies to high frequencies. See $\$ 7.2$ for a discussion of how this is done to enable the proofs in [14,17.

\section{LINEAR DYNAMICS IN DIMENSION $d=3$}

4.1. Coordinate transform. In three dimensions, the vorticity formulation seems less advantageous (especially for the nonlinear problem; see 11 for a brief discussion). One reason for this is that, unlike the $2 \mathrm{D}$ case in (2.2), the linear evolution of the vorticity is not a passive transport equation but instead also involves a nonlocal term giving rise to vortex stretching. Even in the limit $t \rightarrow \infty$, it is not true that the dominant dynamics are passive scalar transport uniformly in $\nu$. Nevertheless, 
it will still be advantageous to begin by factoring out by the transport as in (2.3). Hence, we set

$$
\left\{\begin{array}{l}
X=x-t y \\
Y=y \\
Z=z
\end{array}\right.
$$

As above, we capitalize the physical variables taken in this new set of coordinates: $U(X, Y, Z)=u(x, y, z)$, and denote $\nabla_{L}=\left(\begin{array}{c}\partial_{X} \\ \partial_{Y}-t \partial_{X} \\ \partial_{Z}\end{array}\right)$ and $\Delta_{L}=\nabla_{L} \cdot \nabla_{L}$ for the differential operators in the new coordinates. In these new coordinates, the equation satisfied by $U$ reads

$$
\left\{\begin{array}{l}
\partial_{t} U+U \cdot \nabla_{L} U=\left(\begin{array}{c}
-U^{2} \\
0 \\
0
\end{array}\right)-\nabla_{L} P+\nu \Delta_{L} U, \\
\nabla_{L} \cdot U=0 \\
U(t=0)=U_{\mathrm{in} .}
\end{array}\right.
$$

Linearizing this for small perturbations gives the system

$$
\left\{\begin{array}{l}
\partial_{t} U=\left(\begin{array}{c}
-U^{2} \\
0 \\
0
\end{array}\right)-\nabla_{L} P+\nu \Delta_{L} U, \\
\nabla_{L} \cdot U=0 \\
U(t=0)=U_{\mathrm{in} .}
\end{array}\right.
$$

Notice that in (4.2) the pressure is given by

$$
\Delta_{L} P=-2 \partial_{x} U^{2} \text {. }
$$

4.2. Inviscid damping and enhanced dissipation of $U^{2}$. As it happens, inviscid damping is still relevant in $3 \mathrm{D}$, despite the vortex stretching. Interestingly, [11] is the first work known to the authors that explicitly points out the relevance of inviscid damping to 3D fluid mechanics. This is likely because it, at first, appears to be a relatively minor detail in the overall physical picture of the dynamics. However, as discussed below in Remark 5.1, the presence of inviscid damping will be absolutely critical to the proof of the results in [11-13]. In order to see the effect in $3 \mathrm{D}$, we introduce a new variable, $Q^{2}=\Delta_{L} U^{2}$. This unknown is well known in the hydrodynamic stability of shear flows (see, e.g., 107]) and was apparently introduced by Kelvin [70] (more precisely, the unknown $q^{2}=\Delta u^{2}$ is normally the unknown studied). A small computation reveals that $Q^{2}$ solves

$$
\partial_{t} Q^{2}=\nu \Delta_{L} Q^{2}
$$

(this calculation is actually easier to carry out in $(x, y, z)$ and then transfer to $(X, Y, Z)$ afterward). Since

$$
U^{2}(t)=\Delta_{L}^{-1} Q^{2}(t),
$$

we derive the following, which is independent of Reynolds number and is analogous to (2.7):

$$
\left\|U_{\neq}^{2}(t)\right\|_{H^{s}} \lesssim \frac{1}{\langle t\rangle^{2}}\left\|Q_{i n}^{2}\right\|_{H^{s+2}}
$$


Similarly, the Orr mechanism manifests on $U^{2}$ similar to the way it manifests in 2D. By an analogous computation using (4.3), we also have the rapid decay of the pressure

$$
\|P(t)\|_{H^{s}} \lesssim \frac{1}{\langle t\rangle^{4}}\left\|\Omega_{i n}\right\|_{H^{s+4}}
$$

Finally, since (4.4) is the same as (2.6), it is clear that $Q^{2}$ experiences the same enhanced dissipation effect as $\Omega$ does in $2 \mathrm{D}$ (see $\$ 2.2$ ).

4.3. Vortex stretching and enhanced dissipation of $U^{1,3}$. From 4.5 and (4.6), we see that the $P$ and $U^{2}$ terms in

$$
\begin{aligned}
\partial_{t}\left(\begin{array}{c}
U^{1} \\
U^{3}
\end{array}\right) & =\left(\begin{array}{c}
-U^{2} \\
0
\end{array}\right)-\left(\begin{array}{c}
\partial_{X} P \\
\partial_{Z} P
\end{array}\right)+\left(\begin{array}{c}
\nu \Delta_{L} U^{1} \\
\nu \Delta_{L} U^{3}
\end{array}\right), \\
0 & =\partial_{X} U^{1}+\left(\partial_{Y}-t \partial_{X}\right) U^{2}+\partial_{Z} U^{3},
\end{aligned}
$$

decay rapidly on $O(1)$ time-scales $(O(1)$ relative to the Reynolds number). It is therefore acceptable to choose $u_{i n}^{2}=0$ (this does not alter the generic behavior), for which on time-scales $1 \lesssim t$, the above becomes

$$
\begin{aligned}
\partial_{t}\left(\begin{array}{l}
U^{1} \\
U^{3}
\end{array}\right) & =\left(\begin{array}{l}
\nu \Delta_{L} U^{1} \\
\nu \Delta_{L} U^{3}
\end{array}\right), \\
\partial_{X} U^{1}+\partial_{Z} U^{3} & =0 .
\end{aligned}
$$

There are two main observations here: (A) $U_{\neq}^{1}$ and $U_{\neq}^{3}$ experience the same enhanced dissipation as $U^{2}$, but (B) they do not experience any inviscid damping. In fact, for time-scales $1 \ll t \ll \nu^{-1 / 3}$, the definition of the coordinate transform gives that for some fixed, time-independent $U_{\infty}^{1,3}$,

$$
\begin{aligned}
& u^{1}(t, x, y, z) \sim U_{\infty}^{1}(x-t y, y, z), \\
& u^{3}(t, x, y, z) \sim U_{\infty}^{3}(x-t y, y, z)
\end{aligned}
$$

see [11. Hence, in general, the vorticity $\omega=\nabla \times u$ grows (e.g., in $L^{2}$ ) linearly in time as $O(\epsilon\langle t\rangle)$ until the dissipation time-scale $\nu^{-1 / 3}$ and $u^{1,3}$ experience a matching linear-in-time kinetic energy cascade. This vortex stretching adds extra complexity to both the proofs and the actual nonlinear dynamics in $3 \mathrm{D}$ vs $2 \mathrm{D}$ (for example, see the complexity difference between the $2 \mathrm{D}$ work [18] and the analogous $3 \mathrm{D}$ work [13]). Moreover, the Orr mechanism in 3D not only involves the unmixing of enstrophy from small-to-large scales as originally observed by Orr, but now also involves a more subtle vorticity stretching effect which is anisotropic in $(X, Y)$ frequencies (see, e.g., 98). This will ensure that the 3D resonances are significantly more complicated than $2 \mathrm{D}$.

4.4. The lift-up effect. There is an additional linear effect that was discovered in [46] (see also [74] for extensions to low wave numbers in $x$ ). This effect is active for $x$-independent modes $\left(U_{0}\right)$ : for these modes, $\nabla_{L}=\nabla$ and $\Delta_{L}=\Delta$, so that the linearization is given by

$$
\partial_{t} U_{0}=\left(\begin{array}{c}
-U_{0}^{2} \\
0 \\
0
\end{array}\right)+\nu \Delta U_{0} .
$$


Fortunately, this system can be solved explicitly:

$$
\left\{\begin{aligned}
U_{0}^{1}(t) & =e^{\nu t \Delta}\left[U_{\mathrm{in}, 0}^{1}-t U_{i n, 0}^{2}\right], \\
U_{0}^{2}(t) & =e^{\nu t \Delta} U_{\mathrm{in}, 0}^{2} \\
U_{0}^{3}(t) & =e^{\nu t \Delta} U_{\mathrm{in}, 0}^{3} .
\end{aligned}\right.
$$

This means that $u^{1}$ undergoes a linear growth until time $\sim \frac{1}{\nu}$, at which point the viscosity will begin to slowly relax $u^{1}$ to zero. In fact, the dynamics are essentially the same as the canonical nonnormal ODE system discussed in Section 1.2

$$
\partial_{t}\left(\begin{array}{l}
X_{1} \\
X_{2}
\end{array}\right)=\left(\begin{array}{c}
-\nu X_{1}-X_{2} \\
-\nu X_{2}
\end{array}\right) .
$$

This transient growth is called the lift-up effect and is one of the main destabilizing mechanisms in 3D (it is absent in $2 \mathrm{D}$ since there the divergence-free condition imposes $U_{0}^{2}=0$ ). This instability is one of the primary culprits for subcritical transition in $3 \mathrm{D}$, and it implies that $3 \mathrm{D}$ flows generally have very different dynamics from their $2 \mathrm{D}$ counterparts. For example, one can easily verify that every nontrivial 3D shear flow undergoes this transient growth (this is easily seen on the 3D Euler equations, $\nu=0$, where the lift-up effect becomes an unbounded, linear-intime algebraic instability). The lift-up effect and the nonlinear effects of vorticity stretching together imply that, at high Reynolds numbers, the hydrodynamic stability of $2 \mathrm{D}$ shear flows is very different from that of 3D shear flows (consistent with experimental observations).

\section{NONLINEAR DyNAMiCS IN DIMENSiON THREE}

5.1. Streaks. Observe that for $u_{E}(y)=(y, 0,0)^{t}$, if the initial data in (1.1) is independent of $x$, then this remains true for all time: $u(t, x, y, z)=u(t, y, z)$. In fact, $u^{2,3}(t)$ solves the $2 \mathrm{D}$ Navier-Stokes equations in $(y, z) \in \mathbb{R} \times \mathbb{T}$, whereas $u^{1}$ solves the linear advection-diffusion equation,

$$
\partial_{t} u^{1}+\left(u^{2} \partial_{y}+u^{3} \partial_{z}\right) u^{1}=-u^{2}+\nu \Delta u^{1} .
$$

(Solutions to the Navier-Stokes equations of this type are generally called "2.5 dimensional"; see, e.g., 87].) We will refer to these solutions as streaks. Due to the lift-up effect, the vast majority of the kinetic energy of a streak is in $u^{1}$ [101]; in experiments and computer simulations this, together with the low wave number in $x$, gives them a very distinct streaky appearance [24,72,101,107] (for $x \in \mathbb{R}$, the energy is piling into smaller and smaller wave numbers in $x$; see [74). Using the lift-up effect, it is easy to construct streaks which are initially $O(\nu)$ in $L^{2}$ but at $t \approx \nu^{-1}$, satisfy $\left\|u^{1}(t)\right\|_{L^{2}} \gtrsim 1$. As solutions to the 2D Navier-Stokes equations are global in time, this is a class of global solutions to the 3D Navier-Stokes equations which are from equilibrium relative to $\nu$.

If one only considers the linear problem, then recall that $U_{\neq}$is dissipated on the short time-scale $\nu^{-1 / 3}$ so that streaks are potentially metastable states of the $3 \mathrm{D}$ nonlinear problem that are somewhat analogous to the shear flows in 2D. There are some important differences however. For example, due to the vortex stretching, the velocity field is far from the streak solutions in $L^{2}$ for $t \ll \nu^{-1 / 3}$, whereas in $2 \mathrm{D}$, the inviscid damping ensures that the velocity is close to a shear flow after times which are $O(1)$ relative to $\nu$ (a difference with major nonlinear implications). Assuming that the solution does not transition to a turbulent state, for $t \gg \frac{1}{\nu}$, dissipation in the $k=0$ modes send $U_{0}$ to zero. 
5.2. A scenario for subcritical transition. If streaks are metastable states near the stability threshold, a possible storyline emerges:

- Start at $t=0$ with a perturbation $u_{\text {in }}$ of size $\sim \epsilon$.

- At times $t \gg \frac{1}{\nu^{1 / 3}}$, enhanced dissipation kicks in and dissipates $U_{\neq}$. As a result, the solution looks essentially like a streak.

- At time $t \sim \frac{1}{\nu}$, the maximal linear growth from the lift-up effect predicts $U_{0}^{1}$ could have size $\sim \frac{\epsilon}{\nu}$.

- There are now two possibilities:

- If $\frac{\epsilon}{\nu} \ll 1$, the maximal size of the streak is $\frac{\epsilon}{\nu}$ and the dissipation of modes $k=0$ should, for times $t \gg \frac{1}{\nu}$, bring $u$ back to zero without transitioning to a fully nonlinear state.

- If $\frac{\epsilon}{\nu} \gg 1$, we eventually leave the perturbative regime, and additional instabilities should be expected at around the time $\epsilon^{-1}$, when the streak becomes $O(1)$.

If this storyline is accurate, it would lead to two conclusions: we could determine the stability threshold is precisely $\gamma=1$ and we would have that all instabilities would result from the secondary instability of a streak. That is, a streak grows such that $\left\|u_{0}^{1}\right\|_{L^{2}}=O(1)$, and then the slowly varying shear flow $\left(y+u_{0}^{1}(t), 0,0\right)$ develops a true exponential instability; e.g., if one formally freezes $t$ [101, then one can (formally) create an inviscid, inflection-point shear flow instability. Such an instability would develop very rapidly relative to the time-scales on which the streak varies, and carry the solution rapidly into a strongly nonlinear regime (though it is not quite accurate to suggest it will necessarily go straight into a turbulent state; see [44] and the references therein for more details on this). This secondary instability is called streak breakdown in the fluid mechanics literature, and it has attracted a lot of attention as it is the scenario most often observed in computer simulations and physical experiments; see [24, 32, 45, 101, 107, 114] and the references therein.

The works 11, 12 together confirm at least the beginning of this general picture in Gevrey- $s^{-1}$ with $s>1 / 2$, that is $\left\|e^{\lambda\langle\nabla\rangle^{s}} u_{\text {in }}\right\|_{2}=\epsilon$ and for $\epsilon \lesssim \nu^{2 / 3+\delta}$ for any small $\delta>0$. Note this latter condition is simply a requirement that the data is not "too far" above the stability threshold; it is obvious we need an assumption of this general form, however it is an open question as to whether $2 / 3$ should be the sharp exponent. The two conditions are used to deduce that fully $3 \mathrm{D}$ nonlinear effects are dominated by the enhanced dissipation.

5.3. Null forms and nonlinear interactions. The scenario that was just proposed is relevant if the (mostly linear) heuristics which were derived in the previous paragraphs apply. This can only be the case if nonlinear effects remain small. One reason why this is the case in the regimes we consider goes by the name of null form. The idea is the following: the most potentially threatening nonlinear interactions between linear waves are ruled out by the particular structure of the equation. These weakly nonlinear interactions are significantly more complicated in 3D than the 2D echoes discussed in $\$ 3.2$ However, the nonlinear structure of the 3D Navier-Stokes equations near Couette flow is still very specific, and we can 
recognize a few special structures. For example, here are a few basic observations (not at all exhaustive):

(a) Self-interactions of the lift-up effect. Recall that $U_{0}^{1}$ grows linearly until time $\sim \frac{1}{\nu}$. A quadratic interaction of $U_{0}^{1}$ with itself would be catastrophic, but it is not allowed by the nonlinearity of the equation.

(b) Inviscid damping vs. convection. Recall that the convection term above in (4.1) reads, when spelled out

$$
U \cdot \nabla_{L} U=\left[U^{1} \partial_{X}+U^{2}\left(\partial_{Y}-t \partial_{X}\right)+U^{3} \partial_{Z}\right] U
$$

The second summand, $U^{2}\left(\partial_{Y}-t \partial_{X}\right) U$, seems quite threatening, since it contains a linearly growing factor. Fortunately, it is paired with $U^{2}$ which, as we saw in Section 4.2, is damped independently of Reynolds number via inviscid damping. A similar observation also damps problematic terms in the pressure as a similar $U^{2}\left(\partial_{Y}-t \partial_{X}\right) U^{j}$ structure is preserved therein as well.

Remark 5.1. Note that observation (b) emphasizes the key relevance of inviscid damping for understanding the nonlinear problem in 3D. Indeed, inviscid damping might look like a minor detail in the statements of our results 11 13 or in the general physical picture of the dynamics laid out above. However, inviscid damping plays a crucial role in the proof due to this kind of nonlinear structure.

There are several nonlinear mechanisms which have the potential to cause instability, and many have been proposed as important in the applied mathematics and physics literature for understanding transition; see, e.g., 34, 101, 107, 114 and the references therein. We are particularly worried about so-called bootstrap mechanisms [14, 113, 114, 116, 117, 119]: nonlinear interactions that repeatedly excite growing linear modes. We will classify the main effects by the $x$ frequency of the interacting functions: denote for instance $0 \cdot \neq \rightarrow \neq$ for the interaction of a zero mode (in $x$ ) and a nonzero mode (in $x$ ) giving a nonzero mode (in $x$ ), and similarly, with obvious notations, $0 \cdot 0 \rightarrow 0, \neq \cdot \neq \rightarrow \neq$, and $\neq \cdot \neq \rightarrow 0$.

- $0 \cdot 0 \rightarrow 0$ : These correspond to self-interactions within the streak.

- $0 \cdot \neq \rightarrow \neq$ : These interactions are essentially those that arise when linearizing an $x$-dependent perturbation of a streak and so are connected to the secondary instabilities observed in larger streaks [32,101]. The instabilities which are most commonly observed in experiments are generally related to secondary linear instabilities of the streaks, and so it is unsurprising that most of the leading order interactions are of this type.

- $\neq \cdot \neq \rightarrow \neq$ : These effects include the $3 \mathrm{D}$ variants of the $2 \mathrm{D}$ hydrodynamic echo phenomenon as observed in [130, 131]: nonlinear interactions of $x$ dependent modes forcing unmixing modes [14, 92, 116] - a nonlinear manifestation of the Orr mechanism. In 3D, this is still important, and the range of possible interactions is much wider; see, e.g., 34, 107, 126].

- $\neq \cdot \neq \rightarrow 0$ : These effects are the nonlinear feedback from $x$-dependent modes back into $x$-independent modes.

All of these interactions are coupled to one another, and one can imagine bootstrap mechanisms involving several of them (e.g., $u_{0}^{1}$ forces a nonzero mode which unmixes and then strongly forces $u_{0}^{2}$ which strongly forces $u_{0}^{1}$ via the lift-up effect, and repeat). 
Toy models such as that derived in 33.2 can be written down for $3 \mathrm{D}$; however, it is not really practical to simultaneously include all of the leading order interactions in the same model. Instead, in [11,12 we were content with deriving a much rougher toy model meant only to help provide upper bounds on the nonlinear resonances. Even then, the toy models are $6 \times 6$ ODEs involving many terms which contain information involving all of the linear dynamics deduced above.

\section{Statement of the theorems}

For the sake of simplicity, we focus in this section on results in Gevrey regularity, and we only give short statements of a selection of theorems, referring the interested reader to the original papers.

\subsection{The case of dimension 2 .}

Theorem 6.1 (Stability of Couette flow in dimension 2 [14, [17]). Consider (NS) for $\nu>0$, or $(\mathrm{E})$, in which case $\nu=0$. Fix $s \in\left(\frac{1}{2}, 1\right)$ and $\lambda_{0}>\lambda^{\prime}>0$. Then, if

$$
\left\|u_{\text {in }}\right\|_{\mathcal{G}^{\lambda ; s}}=\epsilon
$$

is sufficiently small (depending only on $s, \lambda_{0}, \lambda^{\prime}$ ), then the unique, classical solution $u(t)$ to (1.1) with initial data $u_{\text {in }}$ is global in time and enjoys the following estimates (all implicit constants might depend on $s, \lambda_{0}, \lambda^{\prime}$, but not on $\nu$ ):

(i) inviscid damping and enhanced dissipation of the velocity field,

$$
\left\|u_{\neq}^{1}(t)\right\|_{L^{2}}+\langle t\rangle\left\|u^{2}(t)\right\|_{L^{2}} \lesssim \frac{\epsilon}{\langle t\rangle\left\langle\nu t^{3}\right\rangle^{10}} ;
$$

(ii) enhanced dissipation on time scales $\gtrsim \frac{1}{\nu^{1 / 3}}$,

$$
\left\|\omega_{\neq}(t, x+t y+t \psi(t, y))\right\|_{\mathcal{G}^{\lambda^{\prime} ; s}} \lesssim \frac{\epsilon}{\left\langle\nu t^{3}\right\rangle^{10}}
$$

(here $\psi(t, y)$ is an $O(\epsilon)$ correction to the mixing which depends on the disturbance);

(iii) classical viscous decay of the zero modes

$$
\left\|u_{0}\right\|_{L^{2}} \lesssim \frac{\epsilon}{\langle\nu t\rangle^{1 / 4}}
$$

Remark 6.2. One can also make more precise the metastability assertions in $\$ 3.1$ by showing the existence of a shear flow $u_{\infty}=\left(u_{\infty}^{1}(y), 0\right)$ such that $u(t) \approx u_{\infty}$ on timescales $1 \ll t \ll \nu^{-1 / 3}$. Similarly, one can prove the existence of an $\omega_{\infty}=\omega_{\infty}(x, y)$ such that $\omega(t, x, y) \approx \omega_{\infty}(t, x+t y+t \psi(t, y))$ for times $1 \ll 1 \ll \nu^{-1 / 3}$, which is analogous to scattering in dispersive equations; see [14,17] for more discussions.

To summarize, the above theorem states that the set of shear flows is asymptotically stable in Gevrey topology in the Euler equations (in a sense), and, uniformly in $\nu>0$, in the Navier-Stokes equations. When viscosity is turned on, an additional effect appears, namely enhanced dissipation, but this is a stabilizing mechanism, and hence the result is uniform in $\nu$. 


\subsection{The case of dimension 3 .}

Theorem 6.3 (Below threshold dynamics [1]). Fix $s \in(1 / 2,1)$, and let $\lambda_{0}>\lambda^{\prime}>$ 0 . Then there exists $c_{0}$ such that if

$$
\left\|u_{\mathrm{in}}\right\|_{\mathcal{G}^{\lambda ; s}}=\epsilon<c_{0} \nu
$$

then the unique, classical solution $u(t)$ to (1.1) with initial data $u_{\text {in }}$ is global in time and enjoys the following estimates (all implicit constants might depend on $s, \lambda_{0}$, $\lambda^{\prime}$, but not on $\nu$ ):

(i) the rapid convergence to a streak through enhanced dissipation,

$$
\left\|u_{\neq}(t)\right\|_{L^{2}} \lesssim \frac{\epsilon\langle t\rangle^{\frac{1}{10}}}{\left\langle\nu t^{3}\right\rangle^{10}}
$$

(the exponents $\frac{1}{10}$ and 10 are not sharp and are only here to fix ideas);

(ii) transient growth of the streak for $t<\frac{1}{\nu}$ through the lift-up effect,

$$
\begin{aligned}
&\left\|u_{0}^{1}(t)-\left(e^{\nu t \Delta}\left(u_{\text {in } 0}^{1}-t u_{\text {in } 0}^{2}\right)\right)\right\|_{\mathcal{G}^{\lambda^{\prime} ; s}} \lesssim\left(\frac{\epsilon}{\nu}\right)^{2}, \\
&\left\|u_{0}^{2}(t)-e^{\nu t \Delta} u_{\text {in } 0}^{2}\right\|_{\mathcal{G}^{\lambda^{\prime} ; s}}+\left\|u_{0}^{3}(t)-e^{\nu t \Delta} u_{\text {in } 0}^{3}\right\|_{\mathcal{G}^{\lambda^{\prime} ; s}} \lesssim\left(\frac{\epsilon}{\nu}\right) \epsilon ;
\end{aligned}
$$

(iii) decay of the background streak for $t>\frac{1}{\nu}$,

$$
\|u(t)\|_{\mathcal{G}^{\lambda^{\prime} ; s}} \lesssim \frac{\epsilon}{\nu\langle\nu t\rangle^{1 / 4}}
$$

In particular, 6.3a (together with the other estimates) show that it is possible to find initial data of the size $\epsilon=c_{0} \nu$ but such that at some finite time $t_{\star} \approx \nu^{-1}$, $\left\|u_{0}^{1}(t)\right\|_{L^{2}} \gtrsim c_{0}$. Hence, one has arbitrarily small solutions which become $O(1)$ with respect to the Reynolds number. However, these solutions are not quite large enough to trigger transition, as is evident by the fact that all the estimates in Theorem 6.3 continue until $t \rightarrow \infty$.

Next, one is interested in studying the dynamics of solutions above the stability threshold. This is the content of the next theorem.

Theorem 6.4 (Above threshold dynamics [12]). Fix $s \in(1 / 2,1)$, and let $\lambda_{0}>$ $\lambda^{\prime}>0$. Then, for all $\delta>0$ all $\nu$ and $c_{0}$ sufficiently small (depending only on $\left.s, \lambda_{0}, \lambda^{\prime}, \delta\right)$, if

$$
\left\|u_{\text {in }}\right\|_{\mathcal{G}^{\lambda ; s}}=\epsilon<\nu^{2 / 3-\delta}
$$

then the unique, classical solution $u(t)$ to (1.1) with initial data $u_{\text {in }}$ exists at least until time $T_{F}=c_{0} \epsilon^{-1}$ and enjoys the following estimates with all implicit constants independent of $\nu, \epsilon, c_{0}$ and $t$ :

(i) the rapid convergence to a streak through enhanced dissipation,

$$
\left\|u_{\neq}(t)\right\|_{L^{2}} \lesssim \frac{\epsilon t^{\frac{1}{10}}}{\left\langle\nu t^{3}\right\rangle^{10}} ;
$$

(ii) transient growth of the streak for $t<T_{F}$ through the lift-up effect,

$$
\begin{aligned}
&\left\|u_{0}^{1}(t)-e^{\nu t \Delta}\left(u_{i n 0}^{1}-t u_{i n 0}^{2}\right)\right\|_{\mathcal{G}^{\lambda^{\prime} ; s}} \lesssim c_{0}^{2}, \\
&\left\|u_{0}^{2}(t)-e^{\nu t \Delta} u_{i n 0}^{2}\right\|_{\mathcal{G}^{\lambda^{\prime} ; s}}+\left\|u_{0}^{3}(t)-e^{\nu t \Delta} u_{i n 0}^{3}\right\|_{\mathcal{G}^{\lambda^{\prime} ; s}} \lesssim c_{0} \epsilon ;
\end{aligned}
$$


(iii) uniform control of the background streak for $t<T_{F}$,

$$
\frac{1}{\langle t\rangle}\left\|u_{0}^{1}(t)\right\|_{\mathcal{G}^{\lambda^{\prime} ; s}}+\left\|u_{0}^{2}(t)\right\|_{\mathcal{G}^{\lambda^{\prime} ; s}}+\left\|u_{0}^{3}(t)\right\|_{\mathcal{G}^{\lambda^{\prime} ; s}} \lesssim \epsilon,
$$

where $\psi(t, y, z)$ is an $O(\epsilon t)$ correction to the mixing which depends on the disturbance.

The key observation above is that Theorem 6.4 shows that near the transition threshold, the only possible instability is the secondary instability of a streak as envisioned in [32,101,114] and others.

\section{Rigorous mathematical proofs}

7.1. Nonlinear change of coordinates. We saw in Sections 2.1 and 4.1 that a change of variable was necessary to take into account the effect of convection by the Couette flow in the linearized equation.

7.1.1. The 2D case. In the work [18, the viscosity is (barely) large enough to imply that the viscous effects dominate the adjustments to the shear flow over long times, and hence in [18, (2.3) is essentially sufficient. However, in [14, 17] the viscosity is nonexistent or arbitrarily small relative to the size of the perturbation (respectively), and hence, the perturbation to the shear cannot be neglected. In these cases, we use the following ansatz with a function $\psi$ to be determined:

$$
\begin{aligned}
& X=x-t y-t \psi(t, y), \\
& Y=y+\psi(t, y) .
\end{aligned}
$$

The purpose of $x \mapsto X$ is to account for the mixing due to the background shear flow and, equivalently, to find a coordinate system in which the vorticity could have uniform-in-time higher regularity estimates. The purpose of $y \mapsto Y$ is so that $\partial_{y} \mapsto\left(1+\partial_{y} \psi\right)\left(\partial_{Y}-t \partial_{X}\right)$, which implies that the critical times still occur at $t \sim \eta / k$ (see (7.5) and [14]). This ansatz is applied, and we derive

$$
\begin{aligned}
\partial_{t} \Omega & +\left(\begin{array}{c}
U^{1}-\partial_{t}(t \psi) \\
U^{2}+\partial_{t} \psi-\nu \partial_{y y} \psi
\end{array}\right) \cdot\left(\begin{array}{c}
\partial_{X} \Omega \\
\left(1+\partial_{y} \psi\right)\left(\partial_{Y}-t \partial_{X}\right) \Omega
\end{array}\right) \\
& =\nu \partial_{X X} \Omega+\nu\left(1+\partial_{y} \psi\right)^{2}\left(\partial_{Y}-t \partial_{X}\right)^{2} \Omega .
\end{aligned}
$$

Note that $\psi$ and $u$ are still in $(x, y)$ coordinates. If one assumes that the linear problem is a good approximation for the dynamics, then $u_{0}^{1}$ is the only contribution to the drift which is not decaying quickly. Hence, it makes sense to choose $\psi$ in order to eliminate this, which is the choice made in [14,17]:

$$
\left\{\begin{array}{l}
\partial_{t}(t \psi)=u_{0}^{1}+\nu \partial_{y y}(t \psi) \\
\lim _{t \searrow 0}(t \psi)=0
\end{array}\right.
$$

After applying this choice and using $U^{1}=-\left(1+\partial_{y} \psi\right)\left(\partial_{Y}-t \partial_{X}\right) \Phi$ and $U^{2}=\partial_{X} \Phi$, (7.2) becomes

$$
\left\{\begin{array}{l}
\partial_{t} \Omega+g \partial_{Y} \Omega+\left(1+\partial_{y} \psi\right) \nabla^{\perp} \Phi_{\neq} \cdot \nabla \Omega=\nu \partial_{X X} \Omega+\nu\left(1+\partial_{y} \psi\right)^{2}\left(\partial_{Y}-t \partial_{X}\right)^{2} \Omega \\
\Delta_{t} \Phi:=\partial_{X X} \Phi+\left(1+\partial_{y} \psi\right)^{2}\left(\partial_{Y}-t \partial_{X}\right)^{2} \Phi+\partial_{y y} \psi \partial_{y} \psi\left(\partial_{Y}-t \partial_{X}\right) \Phi=\Omega, \\
g=\partial_{t} \psi-\nu \partial_{y y} \psi=\frac{1}{t}\left(u_{0}^{1}-\psi\right) .
\end{array}\right.
$$

Notice the crucial cancellation that eliminated powers of $t$ from the nonlinearity; such a nice structure is not present in 3D. The key difference between (7.4) and 
(2.5), is that the velocity field contains only $\Phi_{\neq}$, rather than $\Phi$ : the drift due to the slowly decaying shear flow has been removed from the equation for $\Omega$. The presence of this perturbation is now being felt indirectly through $\psi$ via the forcing term (7.3) (in particular, note that $\psi$ is an unknown that must be solved for along with $\Omega$ ). One can liken the coordinate transform (7.1), (7.3) to a kind of gauge transformation. Note that this coordinate change has made (7.4) significantly more nonlinear. In the proofs of [14, 17, further governing equations are derived for $\Psi^{\prime}(t, Y)=\partial_{y} \psi(t, y)$ and $G(t, Y)=g(t, y)$ in order to obtain estimates on these nonlinear contributions.

Notice that, like $\Delta_{L}, \Delta_{t}$ is not elliptic. Indeed, the symbol of $\Delta_{t}$ (as a pseudodifferential operator),

$$
\sigma\left(\Delta_{t}\right)(Y, k, \eta)=-k^{2}-\left(1+\partial_{y} \psi\right)^{2}(\eta-k t)^{2}+i \partial_{y} \psi \partial_{y y} \psi(\eta-k t),
$$

is degenerate at the frequency $\eta=k t$ - the critical times. As alluded to above, the precise form of the coordinate transform (7.1) was motivated by ensuring that the loss of ellipticity in (7.5) still occurs at the same critical times as the Couette flow. This alone is not sufficient for us to consider $\Delta_{t}$ to be a small perturbation of $\Delta_{L}$; that requires $\partial_{y} \psi$ sufficiently small and a variety of arguments which carefully respect the precise way ellipticity is lost. Many variations of such elliptic estimates have appeared in varying levels of complexity in all of the works on Couette flow 11 14, 17, 18. Related arguments also arise when studying the enhanced dissipation due to the dissipative terms on the left-hand side of (7.4).

7.1.2. The $3 \mathrm{D}$ case. In dimension 3 , the lift-up effect is so strong that the coordinate transform ends up being much larger, and we need to account for it via a refined coordinate transform. In [11,13, the following ansatz is made in analogy with that made above:

$$
\left\{\begin{array}{l}
X=x-t y-t \psi(t, y, z) \\
Y=y+t \psi(t, y, z) \\
Z=z
\end{array}\right.
$$

In [12, one also needs to make a more complicated transformation in $Z$ due to the very large size of the streak; see [12] for details. Via a derivation similar to what we applied above, one derives the following PDE for the evolution of $\psi$ :

$$
\frac{d}{d t}(t \psi)+U_{0} \cdot \nabla(t \psi)=u_{0}^{1}-t u_{0}^{2}+\nu \Delta(t \psi)
$$

Notice the nonlinear transport and especially the lift-up effect seen through the presence of $-t u_{0}^{2}$.

7.2. Fourier multiplier norms. One of the main technical tools employed in all of [11 14, 17, 18, as well as [9, 134, 136] are various norms defined via carefully designed Fourier multipliers. For example, in 3D, defining $Q^{i}=\Delta_{L} U^{i}$,

$$
\left\|A^{i}(t, \nabla) Q^{i}\right\|_{L^{2}}=\left(\sum_{k, \ell} \int\left|A^{i}(t, k, \eta, \ell) \widehat{Q^{i}}(t, k, \eta, \ell)\right|^{2} d \eta\right)^{1 / 2}
$$

The multipliers are time-dependent and typically encode two types of estimate: one is $L^{\infty}$ in time (global bound), the other $L^{2}$ in time (dissipation). This follows from 
the simple identity,

$$
\begin{aligned}
\underbrace{\left\|A^{i}(t, \nabla) Q^{i}(t)\right\|_{L^{2}}^{2}}_{\text {uniform bound }} & =\left\|A^{i}(0, \nabla) Q^{i}(0)\right\|_{L^{2}}^{2}+2 \underbrace{\int_{0}^{t}\left\langle\partial_{t} A^{i}(t, \nabla) Q^{i}, A^{i}(t, \nabla) Q^{i}\right\rangle d s}_{\text {dissipation term }} \\
& +\underbrace{2 \int_{0}^{t}\left\langle A^{i}(t, \nabla) \partial_{t} Q^{i}, A^{i}(t, \nabla) Q^{i}\right\rangle d s}_{\text {computed through the equation; absorbed in various ways }}
\end{aligned}
$$

Usually, the multipliers $A^{i}$ are designed as the product of several kinds of Fourier multipliers.

It turns out that a great diversity of Fourier multipliers is needed in the estimates of [11 14, 17, 18, 132] (and also the works in kinetic theory [9, 10]); each work uses a specific, different combination of them. They generally can be classified into the following types:

- Ghost multipliers. These multipliers are those which are uniformly bounded above and below in both $t$ and $\nu$; a Fourier-side analogue of Alinhac's ghost energy method 2]. These do not alter the norm topology and are hence relatively easy to apply. Variants have been applied throughout the works; they have been used in, e.g., 11 13, 18, 132.

- Nonlinear cascade multipliers. These multipliers become weaker in time to allow the solution to lose large amounts of regularity due to a potential frequency cascade. Variants have been used in [9, 11, 12, 14, 17] and have each been designed based on weakly nonlinear toy models such as (3.2). They are much more difficult to use than ghost multipliers, especially those in $12,14,17$.

- Steady loss/gain multipliers. These multipliers steadily become weaker or stronger over long periods of time, for example, the standard Gevrey/analytic regularity multipliers such as $e^{\lambda(t)|\nabla|^{s}}$. However, the works [11 13, 17 . also use a variety of other multipliers which lose or gain in an anisotropic (in frequency and time) way.

- Singular limit multipliers. The most important multiplier in 13 is different. This multiplier is uniformly bounded in $t$ and frequency, but not uniformly in $\nu$. The multiplier was used to estimate the vortex stretching, which is unbounded as $\nu \rightarrow 0$ (but is bounded for all $\nu>0$ ). We have elected to make this a separate classification of multiplier because we believe that such norms have a high probability of being useful in the future.

7.2.1. An example of a ghost multiplier: enhanced dissipation. Consider the simple linearized PDE,

$$
\partial_{t} Q=\nu \Delta_{L} Q
$$

As we saw in Section 2.2, this PDE is easily solved by taking the Fourier transform and integrating. However, once the nonlinearity is added back in, such an approach is not tenable. Indeed, semigroup methods will usually fail as $\nu \rightarrow 0$ since the problem becomes quasi-linear, whereas energy estimates seem a much more promising tool. Therefore, it is very natural to look for an energy method approach to getting enhanced dissipation estimates on (7.7). An approach using multipliers of the type 
we termed "steady loss/gain" was introduced in [17] and adapted in [11, 12, however, while this approach easily yields very strong estimates, it is only suitable for proofs involving high regularity. An alternative approach, which seems to be much simpler and suitable for low regularity, was put forward in 13 (and later applied in [10, 18]). To use this approach, define the multiplier via the linear ODE for $k \neq 0$ :

$$
\begin{aligned}
\frac{\dot{M}}{M} & =-\frac{\nu^{1 / 3}}{\left[\nu^{1 / 3}\left|t-\frac{\eta}{k}\right|\right]^{2}+1}, \\
M(0, k, \eta) & =1 .
\end{aligned}
$$

Notice that there is a constant $c$ (independent of $k, \eta, t$, and $\nu$ ) such that $c<$ $M(t, k, \eta) \leq 1$, and hence this multiplier is of type we termed "ghost multiplier". In particular, its presence does not change a norm:

$$
\left\|M(t, \nabla)\langle\nabla\rangle^{\sigma} f\right\|_{L^{2}} \approx\left\|\langle\nabla\rangle^{\sigma} f\right\|_{L^{2}} .
$$

The crucial property that $M$ satisfies is

$$
1 \lesssim \nu^{-1 / 6}\left(\sqrt{-\dot{M} M(t, k, \eta)}+\nu^{1 / 2}|k, \eta-k t|\right) \quad \text { for } k \neq 0
$$

which implies that

$$
\left\|f_{\neq}\right\|_{L^{2}}^{2} \lesssim \nu^{-1 / 3}\left(\left\|\sqrt{-\dot{M} M} f_{\neq}\right\|_{L^{2}}^{2}+\nu\left\|\nabla_{L} f_{\neq}\right\|_{L^{2}}^{2}\right) .
$$

Now consider the following simple energy estimate on (7.7),

$$
\frac{1}{2} \frac{d}{d t}\left\|M(t, \nabla) Q_{\neq}\right\|_{L^{2}}^{2}=-\left\|\sqrt{-\dot{M} M} Q_{\neq}\right\|_{L^{2}}^{2}-\nu\left\|\nabla_{L} M Q_{\neq}\right\|_{L^{2}}^{2},
$$

hence,

$$
\begin{aligned}
\frac{1}{2} & \left\|M(t, \nabla) Q_{\neq}(T)\right\|_{L^{2}}^{2}+\int_{0}^{T}\left\|\sqrt{-\dot{M} M} Q_{\neq}\right\|_{L^{2}}^{2} d t+\nu \int_{0}^{T}\left\|\nabla_{L} M Q_{\neq}\right\|_{L^{2}}^{2} d t \\
& =\frac{1}{2}\left\|M(0, \nabla) Q_{\neq}(0)\right\|_{L^{2}}^{2} .
\end{aligned}
$$

Applying (7.8) and (7.10) gives us the decay estimate

$$
\int_{0}^{\infty}\left\|M Q_{\neq}(t)\right\|_{L^{2}}^{2} d t \lesssim \nu^{-1 / 3}\|Q(0)\|_{L^{2}}^{2} .
$$

Hence, this estimate scales with the correct $\nu^{-1 / 3}$ characteristic time-scale observed using the Fourier approach. Further, one can adapt this method to obtain exponential decay rates such as $e^{-\delta \nu^{1 / 3} t}$ for sufficiently small $\delta[10$.

7.2.2. An example of a nonlinear cascade multiplier: decay vs. regularity in the simple 2D case. We build here on the toy model derived in Section 3.2 to derive a multiplier which will control the frequency cascade scenario. We focus on the 2D case since the $3 \mathrm{D}$ case is significantly more technical. Recall that in 3.2 , we came up with the simplified model,

$$
\left\{\begin{array}{l}
\partial_{t} \Omega+\nabla^{\perp} \Phi_{H} \cdot \nabla \Omega_{L}=\nu \Delta_{L} \Omega \\
\Delta_{L} \Phi=\Omega
\end{array}\right.
$$


By repeating calculations from $₫ 2.3$, we have the following decay estimate for the effective velocity field due to inviscid damping:

$$
\left\|\nabla^{\perp} \Phi_{\neq}\right\|_{H^{s}} \lesssim \frac{\|\Omega(t)\|_{H^{s+3}}}{\langle t\rangle^{2}}
$$

It follows that if we have a uniform-in-time bound on $\Omega$ in a suitable $H^{s}$ space, then the nonlinearity decays rapidly and the nonlinear dynamics match the linear dynamics predicted by (2.6). The metastability discussed in \$3.1 will also hold accordingly (at least after we quantify the enhanced dissipation in a suitable sense). However, due to the regularity loss in (7.15), it is not clear how to obtain this uniform bound - the loss is far too large even to get a uniform bound in analytic regularity via a Cauchy-Kovalevskaya style argument (even to get $t^{-1}$ decay requires the loss of two derivatives, whereas a Cauchy-Kovalevskaya argument could handle at most the loss of one).

We see that the estimate (17.15) is too simplistic to accomplish anything (though it is optimal if one restricts oneself to standard $H^{s}$ norms and pointwise-in-time estimates). Let us return to (3.2) and try to more precisely estimate the kind of regularity loss that could occur. As we are only interested in an upper bound, we will drop the signs in (3.2). If we assume that $t \sim \eta / k$, then (3.2) simplifies further to the following, assuming that $\Omega_{L}=O(\kappa)$ :

$$
\begin{aligned}
\partial_{t} \widehat{\Omega}_{H}(t, k, \eta) & =\kappa \frac{\eta}{(k-1)^{2}+(\eta-(k-1) t)^{2}} \widehat{\Omega}_{H}(t, k-1, \eta), \\
\partial_{t} \widehat{\Omega}_{H}(t, k-1, \eta) & =\kappa \frac{\eta}{k^{2}+(\eta-k t)^{2}} \widehat{\Omega}_{H}(t, k, \eta) .
\end{aligned}
$$

We will further assume that $\eta \gtrsim k^{2}$ (so that the coefficient in the second equation is large) and that $|t-\eta / k| \lesssim \frac{\eta}{k^{2}}$, which implies that $t$ is near the critical time $\eta / k$ and away from the critical time $\eta /(k-1)$. Applying these simplifications, we derive the toy model of [14]:

$$
\begin{aligned}
\partial_{t} \widehat{\Omega}_{H}(t, k, \eta) & =\kappa \frac{k^{2}}{\eta} \widehat{\Omega}_{H}(t, k-1, \eta), \\
\partial_{t} \widehat{\Omega}_{H}(t, k-1, \eta) & =\kappa \frac{\eta}{k^{2}+(\eta-k t)^{2}} \widehat{\Omega}_{H}(t, k, \eta) .
\end{aligned}
$$

It is possible to find an approximate supersolution of the coupled ODE system (7.16), which, after iterating over all critical times, quantifies precisely how bad the echo cascade can be [14. Next to a critical time $t \sim \frac{\eta}{k}$, the supersolution $w_{C}$ controls the critical frequency $k$, and the supersolution $w_{N C}$ the noncritical frequency $k-1$ :

$$
\begin{aligned}
\left|\Omega_{H}(t, k, \eta)\right| & \lesssim w_{C}(t, k, \eta), \\
\left|\Omega_{H}(t, k-1, \eta)\right| & \lesssim w_{N C}(t, k, \eta),
\end{aligned}
$$

where $w_{C}$ and $w_{N C}$ are functions which satisfy the following (near $t \approx \eta / k$ ):

$$
w_{C}\left(\frac{\eta}{k}+\frac{\eta}{k^{2}}, k, \eta\right) \approx w_{N C}\left(\frac{\eta}{k}+\frac{\eta}{k^{2}}, k, \eta\right) \approx\left(\frac{\eta}{k^{2}}\right)^{1+O(\kappa)} w_{N C}\left(\frac{\eta}{k}-\frac{\eta}{k^{2}} k, \eta\right)
$$

and

$$
\frac{w_{N C}(t, k, \eta)}{w_{C}(t, k, \eta)} \approx \frac{\eta}{k^{2}\left(1+\left|t-\frac{\eta}{k}\right|\right)} .
$$


The first approximate identity shows that the supersolution estimates that the total growth of the two modes is roughly comparable. That is, both the critical and noncritical frequencies grow by a factor of about $O\left(\frac{\eta}{k^{2}}\right)^{c}$ for some fixed $c$. Iterating over all critical times satisfying $\eta \gtrsim k^{2}$ predicts a growth in the mode $(1, \eta)$ by time $t \geq \eta$ like the following (applying Stirling's formula; see [14] for more details):

$$
\left(\frac{\eta^{\sqrt{\eta}}}{(\sqrt{\eta} !)^{2}}\right)^{c} \sim \eta^{-c / 2} e^{2 c \sqrt{\eta}}
$$

Hence, (7.16) precisely predicts that the amount of enstrophy at frequencies comparable to $\eta$ can be amplified by roughly $e^{2 c \sqrt{\eta}}$. This large amplification suggests that Gevrey-2 regularity might be necessary in order to control the nonlinear effects for long times (if one does not have viscosity). This is the origin of the high regularity requirement in [14, 17].

On the other hand the approximate identity (7.17) shows that, very close to $\eta \approx t k$, the noncritical frequencies could potentially be much larger than the critical frequencies. This regularity imbalance is only possible due to the fact that the critical frequency does not interact directly with itself in the leading order dynamics predicted by (7.16). This special noninteraction can be considered a null form which is absolutely crucial to the proof of [14,17] (it arises from the $\nabla^{\perp} \Phi \cdot \nabla$ structure in the original equations). Such null forms, which dictate the precise way information moves between different frequencies, also prove to be essential to the $3 \mathrm{D}$ works, though there the structures are more complicated [11-13].

The proof of [14 is based on a weighted norm roughly of the form $A=$ $e^{\lambda(t)|\nabla|^{s}} \frac{1}{w(t, k, \eta)}$ with $s \in(0,1)$ and $w$ designed to resemble the above supersolution near the critical times $t \sim \eta / j$ for any $j$ with $|j|^{2} \lesssim|\eta|$ (after accounting for the subsequent multiplicative amplification through each critical time). That is, for $k=j, w \sim w_{C}$ and $k \neq j$, we take $w \sim w_{N C}$. The motivation for this choice can be interpreted as follows: $w(t, k, \eta)$ roughly predicts the "worst-case" transfer of information from low-to-high frequencies, and, if the enstrophy transfer is of roughly the same type as that predicted, we can expect that

$$
|\Omega(t, k, \eta)| \lesssim w(t, k, \eta)
$$

which ensures that the norm gets weaker at precisely the rate necessary to ensure $A \Omega$ remains uniformly bounded.

In the 3D works, the analogues of the toy model are far more complicated [11, 12, however, these can be upper bounded by multipliers which are similar to the above $w$. In the work on the Vlasov-Poisson equations, a multiplier analogous to the above $w$ is used, however, it is also a singular limit (in the size of the data $\epsilon$ ), and so it is in some ways more complicated but in other ways simpler, as the resonances in the Vlasov-Poisson equations have a simpler structure [9].

7.2.3. An example of a singular limit multiplier. When deriving estimates, it is natural to define $Q^{1}=\Delta_{L} U^{2}$ and $Q^{3}=\Delta_{L} U^{3}$ in a similar way to $Q^{2}=\Delta_{L} U^{2}$. A caricature of the linearized problem for $Q^{1}$ and $Q^{3}$ is

$$
\partial_{t} Q-\nu \Delta_{L} Q+2 \partial_{X Y} \Delta_{L}^{-1} Q=0 .
$$

Taking the Fourier transform, it reads

$$
\partial_{t} \widehat{Q}+\nu\left(k^{2}+(\eta-k t)^{2}+\ell^{2}\right) \widehat{Q}+2 \frac{k(\eta-k t)}{k^{2}+(\eta-k t)^{2}+\ell^{2}} \widehat{Q}=0 .
$$


This can be seen as a competition between the second term above (dissipation) and the third term (stretching, which causes $Q$ to grow if $k(\eta-k t)<0$ ). In order to quantify how much growth the stretching can generate, define the multiplier $m$ in such a way that first, $m(t=0, \nabla)=1$; and second, if $Q$ satisfies $[7.18$, then

$$
\frac{d}{d t}\|m(t, \nabla) Q\|_{L^{2}} \leq 0 \text {. }
$$

With an appropriate choice of $m$,

$$
\nu^{2 / 3} \lesssim m(t, k, \eta, \ell) \leq 1
$$

(and this bound is sharp). This means that the maximal linear growth which $Q$ can undergo is $\lesssim \nu^{-2 / 3}$. More importantly, the multiplier $m$ will be key in closing nonlinear estimates.

7.3. Paraproducts and Gevrey regularity. The paraproduct, introduced by Bony in 23], has been a ubiquitous tool in the analysis of the stability of the Couette flow. In order to explain its principle, consider functions $f$ and $g$ of a real variable, and recall that the Fourier transform of their product is given by the convolution of their Fourier transforms

$$
\widehat{f g}(\xi)=\int \widehat{f}(\xi-\eta) \widehat{g}(\eta) d \eta .
$$

The idea is now to use a smooth cut-off function $\chi$ such that

$$
\chi(\xi, \eta)= \begin{cases}1 & \text { if }|\eta| \gg|\xi-\eta|, \\ 0 & \text { if }|\xi-\eta| \gg|\eta|\end{cases}
$$

in order to split the product

$$
\widehat{f g}(\xi)=\int \chi(\xi, \eta) \widehat{f}(\xi-\eta) \widehat{g}(\eta) d \eta+\int[1-\chi(\xi, \eta)] \widehat{f}(\xi-\eta) \widehat{g}(\eta) d \eta .
$$

In the right-hand side, the first summand corresponds to interactions between high frequencies of $g$ and low frequencies of $h$. This explains the following shorthand for the above decomposition:

$$
f g=f_{\mathrm{Lo}} g_{\mathrm{Hi}}+f_{\mathrm{Hi}} g_{\mathrm{Lo}} .
$$

In this way the decomposition of all products turns out to be very convenient, in that it allows us to use different estimates for different interactions. Consider for instance the paraproduct decomposition of the convection term

$$
u \cdot \nabla u=u_{\mathrm{Lo}} \cdot(\nabla u)_{\mathrm{Hi}}+u_{\mathrm{Hi}} \cdot(\nabla u)_{\mathrm{Lo}} .
$$

The second term should be better behaved, since the derivative hits low frequencies. This can be quantified through the estimate

$$
\left\|u_{\mathrm{Hi}} \cdot(\nabla u)_{\mathrm{Lo}}\right\|_{H^{s}} \lesssim\|u\|_{H^{s}}^{2} .
$$

As a consequence, this particular term does not lose derivatives, as opposed to the low-high interaction.

Basic paraproduct methods provide a straightforward proof of the product rule for fractional Sobolev spaces (see, e.g., the appendix of [111]), and hence it is reasonable to expect that they also provide a useful tool for deducing product rules in Gevrey class. Indeed, one has the following very useful product rule which emphasizes one of the very convenient properties of Gevrey regularity. Specifically, only one of the two factors is measured in the highest norm in each of the two 
terms (analogous to the situation in Sobolev spaces). We remark that this is false in analytic regularity $s=1$.

Theorem 7.1. Let $s \in(0,1)$, let $p>d / 2$, and let $\lambda>0$. There exists a $c=c(s) \in$ $(0,1)$ depending only on $s$, such that

$$
\|f g\|_{\mathcal{G}^{\lambda, s}} \lesssim_{s, p}\|f\|_{\mathcal{G}^{\lambda, s}}\left\|\langle\nabla\rangle^{p} g\right\|_{\mathcal{G}^{c \lambda, s}}+\|g\|_{\mathcal{G}^{\lambda, s}}\left\|\langle\nabla\rangle^{p} f\right\|_{\mathcal{G}^{c \lambda, s}} .
$$

This theorem can be proved using paraproducts together with elementary inequalities of the general type $|x-y|^{s}<c(s)\left(|x|^{s}+|y|^{s}\right)$ (see, e.g., [14 for a proof). We remark that when working in Gevrey class, it is necessary to split into three regions of frequency rather than two: $|\xi| \ll|\eta-\xi|,|\eta-\xi| \ll|\xi|$, and $|\eta-\xi| \approx|\xi|$.

\section{Related QUESTIONS}

8.1. Scattering and weak turbulence. Scattering is a typical behavior for (defocusing) nonlinear dispersive equations set in the whole space: global solutions look asymptotically linear. To be more specific, consider for instance the nonlinear Schrödinger equation

$$
\left\{\begin{array}{l}
i \partial_{t} \psi-\Delta \psi=-|\psi|^{2} \psi \\
\psi(t=0)=\psi_{0}
\end{array}\right.
$$

set on the whole space $\mathbb{R}^{3}: \psi=\psi(t, x) \in \mathbb{C}$, with $(t, x) \in \mathbb{R} \times \mathbb{R}^{3}$. As $t \rightarrow \pm \infty$, $\psi(t)$ converges locally to zero, and looks increasingly like a linear solution,

$$
\left\|\psi(t)-e^{i t \Delta} f_{ \pm}\right\|_{H^{1}} \rightarrow 0 \quad \text { as } t \rightarrow \pm \infty
$$

(in general, $f_{+}$and $f_{-}$are different).

Recall that, in dimension 2, for $\nu=0$, and for small Gevrey data,

$$
\omega(t, x, y) \approx \omega_{ \pm \infty}\left(t, x-t y-t u_{ \pm \infty}(y), y\right) \quad \text { as } t \rightarrow \pm \infty .
$$

The similarities with scattering for (NLS) are striking: in both cases there is pointwise decay $\left(\psi \rightarrow 0\right.$ and $u^{2} \rightarrow 0$, respectively); the system relaxes as $t \rightarrow \pm \infty$ (to a linear solution), though no dissipation appears in the system, which is fully time-reversible; and the scattering states $\omega_{ \pm \infty}$ differ in general.

The mechanism of relaxation for (NLS) is the spreading of waves, which causes them to decay and ultimately suppresses nonlinear interactions. No such mechanism is available for $(\mathrm{E})$, since the domain is compact in $X$ (and very little spreading in $Y$ occurs). However, the energy is spreading in frequency: the drift toward high frequencies (and the Biot-Savart law) is the cause of inviscid damping.

This is very reminiscent of a phenomenon called weak turbulence. It is expected for (NLS) set on a compact domain, and it consists of a drift of energy toward high frequencies as $t \rightarrow \infty$, causing the system to relax. However, no mathematical evidence is known for this phenomenon, which, furthermore, should be valid in a statistical sense only.

8.2. Landau damping. The Vlasov-Poisson equation is a kinetic model describing the evolution of a density of particles $f(t, x, v)$, which, at time $t$, has position $x$ (usually taken $x \in \mathbb{T}_{x}^{d}$ or $x \in \mathbb{R}_{x}^{d}$ ) and velocity $v \in \mathbb{R}_{v}^{d}$ :

$$
\left\{\begin{array}{l}
\partial_{t} f+v \cdot \nabla_{x} f+E \cdot \nabla_{v} f=0, \\
E(t, x)=\nabla_{x} \Delta_{x}^{-1}\left(\int_{\mathbb{R}^{d}} f d v-\bar{\rho}\right), \\
f(t=0)=f_{\mathrm{in}}
\end{array}\right.
$$


where $\bar{\rho}$ is a neutralizing background density (usually fixed to be a constant) which models the presence of the ions in the plasma [26. At least to start, in physical applications, one studies disturbances of spatially homogeneous background $f(t, x, v)=f^{0}(v)+h(t, x, v)$ with the mean-zero condition $\int_{\mathbb{T}^{d}} \int_{\mathbb{R}^{d}} h(t, x, v) d v d x=0$ and $\bar{\rho}=\int f^{0}(v) d v$. The equations hence become

$$
\left\{\begin{array}{l}
\partial_{t} h+v \cdot \nabla_{x} h+E \cdot\left(\nabla_{v} f^{0}+\nabla_{v} h\right)=0, \\
E=\nabla_{x} \Delta_{x}^{-1} \int_{\mathbb{R}^{d}} h d v \\
h(t=0)=h_{\mathrm{in}}
\end{array}\right.
$$

If $h$ solved the free transport equation with smooth data

$$
\partial_{t} h+v \cdot \nabla_{x} h=0
$$

then it is easy to see that $E \rightarrow 0$ as $t \rightarrow \infty$ (it suffices to take the Fourier transform and proceed exactly as in the Couette flow). This phenomenon is called Landau damping; just like inviscid damping, it gives decay in the absence of a dissipative mechanism. We also remark that it is a variant of velocity averaging in a slightly different guise; see, e.g., [59, 60. In 1946, Landau proved that Landau damping holds for the linearized Vlasov equations on $x \in \mathbb{T}^{d}$ for $f^{0}$ a Maxwellian [75], and Penrose 97 later extended this to a wide class of other backgrounds; see, e.g., 36, 57, 58, 115 for other studies on linearized Vlasov equations. We remark that it was apparently Van Kampen [115] who first pointed out that Landau damping is related to mixing in phase space. It is one of the most fundamental effects in the kinetic theory of plasmas [26, 105] and is also potentially relevant to stellar mechanics [86].

It was first proved that there exist solutions to the nonlinear Vlasov equations (8.2) on $x \in \mathbb{T}$ which display Landau damping by Caglioti and Maffei 27]; later, this was extended further in 67. A major breakthrough came in Mouhot and Villani's work [93], which showed that on $x \in \mathbb{T}^{d}$, the dynamics of all sufficiently small and (sufficiently smooth) Gevrey class solutions matched that of the linearized Vlasov equations for all $t$ (and hence, all such solutions display Landau damping at essentially the same rate as that predicted by Landau and Penrose's work). The proof was later simplified and extended to a wider range of Gevrey regularity by Mouhot and two of the authors in [16], which was later adapted to the relativistic plasma case [129]. In [47,48, Landau damping in Sobolev regularity was proved for variants of the Vlasov-Poisson model which have much weaker resonances (see also [38]). Later, dispersion and phase mixing were combined to prove Landau damping on $\mathbb{R}_{x}^{3} \times \mathbb{R}_{v}^{3}$ in Sobolev regularity for Vlasov-Poisson in [15. In particular, it was proved that dispersive effects are able to limit the effect of the plasma echo resonances. However, in [9], it was proved that on $\mathbb{T}_{x} \times \mathbb{R}_{v}$ there exists solutions arbitrarily close to homogeneous equilibrium in Sobolev spaces which deviate arbitrarily far from the linearized Vlasov predictions due to long chains of plasma echo resonances. Recently in [10], it was shown that nonlinear collisions can suppress these resonances in a manner analogous to the results of [13, 18.

\section{Open PROBLEMS}

The results of [11-14, 17, 18] study what is essentially the easiest problem in hydrodynamic stability at high Reynolds number in the absence of unstable spectra in the linearization. However, now that this case has been studied, it makes sense to 
ask what harder problems can be considered. Moreover, despite the rather in-depth studies, there are still some unanswered questions, even on Couette flow.

9.1. Nonlinear instabilities and transition thresholds: open questions on Couette flow. One of the most important directions to explore further is the role of nonlinear instabilities in subcritical transition, and moreover, how the regularity of initial data may or may not affect the dynamics. In particular, an important problem regarding Couette flow would be to prove the sharpness of the transition thresholds estimated in 2D 18 and 3D 13. for Sobolev regularity data. Similarly, one could evaluate carefully how things change in still lower regularity; for example, see 82 for the 2D Euler equations. At least for the case of the 3D Couette flow, there is a variety of quantitative and anecdotal evidence to suggest that, indeed, regularity does play an important role in the subcritical transition. The numerical studies of Reddy et al. [101] suggest specifically that the threshold in [13] is sharp; however, numerical studies of these problems are very difficult and prone to overestimating the transition thresholds so it is difficult to say confidently either way. It makes sense to try to connect any such analysis to existing formal asymptotic works, such as [116,117] and physical experiments [107, 126]. Finally, one would naturally wish to understand better the secondary instabilities of streaks and the role played in the next step in the transition from nearly linear to nonlinear dynamics.

9.2. Influence of boundaries and low wave numbers. The physical experiments most closely approximating the Couette flow setting are in bounded channels $y \in[-1,1]$ and long channels better modeled by $x \in \mathbb{R}$. The boundary conditions at the top and bottom are naturally $u=(1,0,0)$ at $y=1$ and $u=(-1,0,0)$ at $y=-1$ after proper normalization (we will refer to this case as the infinite channel problem). It has been observed that the presence of physical boundaries could potentially introduce a variety of new kinds of nonlinear instabilities (see [107, 126] and the references therein). Moreover, the presence of long wave numbers in $x$ presents another challenge. It has been proved that many planar shear flows in the channel that are not Couette flow suffer from long-wave instabilities at high Reynolds number; see [50,62 and the references therein. Understanding both (or either) the long wave effects and the boundary effects in 3D is an important goal moving forward. Even at the level of the 2D Euler equations in a channel, it has been observed that boundaries can add new complications [69, 123, 134 .

Remark 9.1. Studying nonlinear stability for the 2D Navier-Stokes equations at high Reynolds number or the $2 \mathrm{D}$ Euler equations in a bounded channel (e.g., $\mathbb{T} \times$ $[-L, L]$ or $\mathbb{R} \times[-L, L])$ may not be of direct physical relevance. As discussed above, real 3D shear flows undergo strong 3D instabilities at high Reynolds number - the $2 \mathrm{D}$ dynamics are rarely observed. Applications of 2D fluid mechanics to plasma physics (via drift-kinetic or gyro-kinetic scenarios) or atmospheric dynamics would generally not involve such boundaries, or, would have boundary conditions (and boundary layers) which are different from the usual no-slip and no-penetration conditions. Nevertheless, this case is very interesting and important to study from a mathematical viewpoint, as one can use this case as an intermediate result on the path to understanding the case of 3D boundaries (which is of high physical interest).

9.3. Stability and subcritical instability for more general problems. A variety of other settings in fluid mechanics are of more direct physical relevance than 
the Couette flow. In two dimensions, the most important configuration to study are vortices in the 2D Navier-Stokes and Euler equations (that is, radially symmetric configurations of vorticity). In analogy with shear flows, for the Euler equations all such configurations are equilibria whereas for the Navier-Stokes equations all such solutions reduce to the heat equation; see [21,54,77] and references therein for progress. In three dimensions there are several configurations of major relevance. Perhaps the most famous of the problems is the pipe flow studied by Reynolds in his original experiments [102]: the equilibrium corresponds to pressure-driven flow in a cylindrical pipe (preferably an infinite pipe, but the periodic model would likely be studied first). Other good examples are wall-bounded shear flows (e.g., semi-infinite domains with a parabolic shear flow above a flat plate), Couette flow in an infinite channel (bounded in $y$ ), or other planar shear flows in an infinite channel [4]. Naturally, one could also study vortex columns. For each of these problems, one can formulate analogues of Questions Q1 and Q2 in various regularities and pursue questions similar to those we studied in the Couette flow. Similarly, various kinds of subcritical instabilities will arise in these problems, and it would be important to isolate them and study them in more detail. Moreover, many similar problems exist in kinetic theory and magneto-hydrodynamics problems, for example, understanding Landau damping in galactic dynamics or in plasmas in the presence of magnetic fields.

As we saw above, the stability of the Couette flow has been analyzed in depth (though some questions remain). One of the important reasons why this was possible is that the linearized problem can be solved explicitly. For more general problems this is no longer possible, and indeed, even the resulting linear problems are extremely difficult to analyze in detail. Linear works studying enhanced dissipation and inviscid damping in the 2D Navier-Stokes and Euler equations have been quite technical and the theory is still in its infancy; see, e.g., [21,54,68, 77, 78, 123, 125, 134, 136. Moreover, our works on Couette flow show that nonlinear results do not easily follow from linear results. However, it is our hope that the ideas and methods which were put forward in the analysis of the Couette flow can lead to a much more general understanding and eventually lay the groundwork for a wider theory.

9.4. Large-time behavior of the Euler equations for $d=2$. The existence of global solutions of the Euler equations (E) in dimension $d=2$ is known under weak assumptions on the data. However, very little is known qualitatively about these solutions. Until recently, the only known solutions whose asymptotic behavior could be established were stationary or explicit solutions! Gevrey solutions close to Couette flow are the first nontrivial solutions whose behavior can be described precisely [14. The works of, e.g., [71, 94] and related norm growth results also provide glimpses into interesting long-term dynamics, though significantly less information is obtained on solutions in these regimes. Moreover, the work of, e.g., [30] constructs a variety of interesting smooth, rigidly rotating solutions. Any solutions of these types clearly do not involve vorticity mixing as $t \rightarrow \infty$, and so we can expect a large set of solutions which do not display any mixing. On the other hand, results of [14] show that all sufficiently smooth solutions in the vicinity of Couette flow which are not shear flows experience some vorticity mixing as $t \rightarrow \infty$ and in particular, are not precompact in $L^{2}(\mathbb{T} \times \mathbb{R})$. In light of [14, statistical mechanics considerations, and a variety of numerical simulations, it is potentially 
reasonable to make the following, somewhat vague, conjecture which suggests that this situation is generic in a suitable sense. This conjecture was first communicated to us by Vladimir Sverak.

Conjecture 9.2. The generic solution to the 2D Euler equations in vorticity form on $\mathbb{T}^{2}$ is such that the orbit $\{\omega(t)\}_{t \in \mathbb{R}}$ is not precompact in $L^{2}\left(\mathbb{T}^{2}\right)$. Here "generic" could be interpreted in the sense of Baire category or in an appropriate probabilistic sense, such as randomizing initial data in a suitable manner.

\section{ABout THE AUTHORS}

Jacob Bedrossian is associate professor of mathematics at University of Maryland, College Park. He obtained his PhD from University of California, Los Angeles in 2011. He works on the analysis of partial differential equations, mostly related to understanding mixing and the stability of coherent structures in fluid mechanics and plasma physics.

Pierre Germain is professor of mathematics at Courant Institute of Mathematical Sciences at New York University. He obtained his PhD from École Polytechnique. He works on harmonic analysis, fluid mechanics, and nonlinear wave equations.

Nader Masmoudi received his degree in mathematics from École Normale Supérieure Paris in 1996, and from Paris Dauphine University both his PhD in 1999 and his HDR in 2000. He works in the area of partial differential equations with applications to fluid mechanics and gas dynamics. He is currently professor at Courant Institute of Mathematical Sciences at New York University (NYU), spending two years at NYU-Abu Dhabi as affiliated faculty.

\section{REFERENCES}

[1] F. Alavyoon, D. S. Henningson, and P. H. Alfredsson, Turbulent spots in plane Poiseuille flow-flow visualization, The Physics of Fluids, 29 (1986), no. 4, 1328-1331.

[2] S. Alinhac, The null condition for quasilinear wave equations in two space dimensions I, Invent. Math. 145 (2001), no. 3, 597-618, DOI 10.1007/s002220100165. MR1856402

[3] V. I. Arnol'd, On conditions for nonlinear stability of plane stationary curvilinear flows of an ideal fluid (Russian), Dokl. Akad. Nauk SSSR 162 (1965), 975-978. MR0180051

[4] J. S. Baggett, T. A. Driscoll, and L. N. Trefethen, A mostly linear model of transition to turbulence, Phys. Fluids 7 (1995), no. 4, 833-838, DOI 10.1063/1.868606. MR.1324952

[5] J. S. Baggett and L. N. Trefethen, Low-dimensional models of subcritical transition to turbulence, Phys. Fluids 9 (1997), no. 4, 1043-1053, DOI 10.1063/1.869199. MR1437563

[6] A. P. Bassom and A. D. Gilbert, The spiral wind-up of vorticity in an inviscid planar vortex, J. Fluid Mech. 371 (1998), 109-140, DOI 10.1017/S0022112098001955. MR.1650153

[7] K. Beauchard and E. Zuazua, Some controllability results for the 2D Kolmogorov equation, Ann. Inst. H. Poincaré Anal. Non Linéaire 26 (2009), no. 5, 1793-1815, DOI 10.1016/j.anihpc.2008.12.005. MR2566710

[8] M. Beck and C. E. Wayne, Metastability and rapid convergence to quasi-stationary bar states for the two-dimensional Navier-Stokes equations, Proc. Roy. Soc. Edinburgh Sect. A 143 (2013), no. 5, 905-927, DOI 10.1017/S0308210511001478. MR3109765

[9] J. Bedrossian, Nonlinear echoes and Landau damping with insufficient regularity, arXiv:1605.06841 (2016).

[10] J. Bedrossian, Suppression of plasma echoes and Landau damping in Sobolev spaces by weak collisions in a Vlasov-Fokker-Planck equation, Ann. PDE 3 (2017), no. 2, Art. 19, 66, DOI 10.1007/s40818-017-0036-6. MR3719106

[11] J. Bedrossian, P. Germain, and N. Masmoudi, Dynamics near the subcritical transition of the 3D Couette flow I: Below threshold, arXiv:1506.03720 (2015); Mem. Amer. Math. Soc. (to appear). 
[12] J. Bedrossian, P. Germain, and N. Masmoudi, Dynamics near the subcritical transition of the 3D Couette flow II: Above threshold, arXiv:1506.03721 (2015).

[13] J. Bedrossian, P. Germain, and N. Masmoudi, On the stability threshold for the 3D Couette flow in Sobolev regularity, Ann. of Math. (2) 185 (2017), no. 2, 541-608, DOI 10.4007/annals.2017.185.2.4. MR3612004

[14] J. Bedrossian and N. Masmoudi, Inviscid damping and the asymptotic stability of planar shear flows in the 2D Euler equations, Publ. Math. de l'IHÉS 122 (2015), no. 1, 195-300.

[15] J. Bedrossian, N. Masmoudi, and C. Mouhot, Landau damping in finite regularity for unconfined systems with screened interactions, Comm. Pure Appl. Math. 71 (2018), no. 3, 537-576, DOI 10.1002/cpa.21730. MR3762277

[16] J. Bedrossian, N. Masmoudi, and C. Mouhot, Landau damping: paraproducts and Gevrey regularity, Ann. PDE 2 (2016), no. 1, Art. 4, 71, DOI 10.1007/s40818-016-0008-2. MR3489904

[17] J. Bedrossian, N. Masmoudi, and V. Vicol, Enhanced dissipation and inviscid damping in the inviscid limit of the Navier-Stokes equations near the two dimensional Couette flow, Arch. Ration. Mech. Anal. 219 (2016), no. 3, 1087-1159, DOI 10.1007/s00205-015-0917-3. MR.3448924

[18] J. Bedrossian, V. Vicol, and F. Wang, The Sobolev stability threshold for $2 D$ shear flows near Couette, arXiv:1604.01831 (2016); J. Nonlin. Sci. (to appear).

[19] J. Bedrossian and M. Coti Zelati, Enhanced dissipation, hypoellipticity, and anomalous small noise inviscid limits in shear flows, Arch. Ration. Mech. Anal. 224 (2017), no. 3, 1161-1204, DOI 10.1007/s00205-017-1099-y. MR.3621820

[20] J. Bedrossian, M. Coti Zelati, and N. Glatt-Holtz, Invariant measures for passive scalars in the small noise inviscid limit, Comm. Math. Phys. 348 (2016), no. 1, 101-127, DOI 10.1007/s00220-016-2758-9. MR3551262

[21] J. Bedrossian, M. C. Zelati, and V. Vicol, Vortex axisymmetrization, inviscid damping, and vorticity depletion in the linearized 2d Euler equations, arXiv:1711.03668 (2017).

[22] A. Bernoff and J. Lingevitch, Rapid relaxation of an axisymmetric vortex, Phys. Fluids, 6 (1994), 3717.

[23] J.-M. Bony, Calcul symbolique et propagation des singularités pour les équations aux dérivées partielles non linéaires (French), Ann. Sci. École Norm. Sup. (4) 14 (1981), no. 2, 209-246. MR631751

[24] S. Bottin, O. Dauchot, F. Daviaud, and P. Manneville, Experimental evidence of streamwise vortices as finite amplitude solutions in transitional plane Couette flow, Physics of Fluids, 10 (1998), 2597.

[25] J. P. Boyd, The continuous spectrum of linear Couette flow with the beta effect, J. Atmospheric Sciences, 40 (1983), no. 9, 2304-2308.

[26] T. J. M. Boyd and J. J. Sanderson, The physics of plasmas, Cambridge University Press, Cambridge, 2003. MR 1960956

[27] E. Caglioti and C. Maffei, Time asymptotics for solutions of Vlasov-Poisson equation in a circle, J. Statist. Phys. 92 (1998), no. 1-2, 301-323, DOI 10.1023/A:1023055905124. MR.1645659

[28] D. R. Carlson, S. E. Widnall, and M. F. Peeters, A flow-visualization study of transition in plane Poiseuille flow, Journal of Fluid Mechanics, 121 (1982), 487-505.

[29] K. M. Case, Stability of inviscid plane Couette flow, Phys. Fluids 3 (1960), 143-148, DOI 10.1063/1.1706010. MR0128230

[30] A. Castro, D. Córdoba, and J. Gómez-Serrano, Uniformly rotating smooth solutions for the incompressible 2d Euler equations, arXiv:1612.08964 (2016).

[31] A. Cerfon, J. Freidberg, F. Parra, and T. Antaya, Analytic fluid theory of beam spiraling in high-intensity cyclotrons, Phys. Rev. ST Accel. Beams, 16 (2013), 024202.

[32] S. J. Chapman, Subcritical transition in channel flows, J. Fluid Mech. 451 (2002), 35-97, DOI 10.1017/S0022112001006255. MR 1886008

[33] P. Constantin, A. Kiselev, L. Ryzhik, and A. Zlatoš, Diffusion and mixing in fluid flow, Ann. of Math. (2) 168 (2008), no. 2, 643-674, DOI 10.4007/annals.2008.168.643. MR2434887

[34] A. D. Craik, Nonlinear resonant instability in boundary layers, Journal of Fluid Mechanics, 50 (1971), no. 2, 393-413.

[35] F. Daviaud, J. Hegseth, and P. Bergé, Subcritical transition to turbulence in plane Couette flow, Phys. Rev. Lett. 69 (1992), no. 17, 2511. 
[36] P. Degond, Spectral theory of the linearized Vlasov-Poisson equation, Trans. Amer. Math. Soc. 294 (1986), no. 2, 435-453, DOI 10.2307/2000192. MR825714

[37] Y. Deng and N. Masmoudi, Long time instability of the Couette flow in low Gevrey spaces, arXiv:1803.01246 (2018).

[38] H. Dietert, Stability and bifurcation for the Kuramoto model (English, with English and French summaries), J. Math. Pures Appl. (9) 105 (2016), no. 4, 451-489, DOI 10.1016/j.matpur.2015.11.001. MR3471147

[39] L. Dikiur, The stability of plane-parallel flows of an ideal fluid, Soviet Physics Doklady 5, (1961), 1179.

[40] P. Drazin and L. Howard, Hydrodynamic stability of parallel flow of inviscid fluid, Advances in Applied Mechanics 9 (1966), 1-89.

[41] P. G. Drazin and W. H. Reid, Hydrodynamic stability, Cambridge Monographs on Mechanics and Applied Mathematics, Cambridge University Press, Cambridge-New York, 1982. MR684214

[42] D. G. Dritschel, Nonlinear stability bounds for inviscid, two-dimensional, parallel or circular flows with monotonic vorticity, and the analogous three-dimensional quasi-geostrophic flows, Journal of Fluid Mechanics 191 (1988), 575-581.

[43] B. Dubrulle and S. Nazarenko, On scaling laws for the transition to turbulence in uniformshear flows, Euro. Phys. Lett. 27 (1994), no. 2, 129.

[44] Y. Duguet, L. Brandt, and B. R. J. Larsson, Towards minimal perturbations in transitional plane Couette flow, Phys. Rev. E (3) 82 (2010), no. 2, 026316, 13, DOI 10.1103/PhysRevE.82.026316. MR2736445

[45] Y. Duguet, P. Schlatter, and D. S. Henningson, Formation of turbulent patterns near the onset of transition in plane Couette flow, J. of Fluid Mech. 650 (2010), 119-129.

[46] T. Ellingsen and E. Palm, Stability of linear flow, Phys. of Fluids 18 (1975), 487.

[47] E. Faou and F. Rousset, Landau damping in Sobolev spaces for the Vlasov-HMF model, Arch. Ration. Mech. Anal. 219 (2016), no. 2, 887-902, DOI 10.1007/s00205-015-0911-9. MR.3437866

[48] B. Fernandez, D. Gérard-Varet, and G. Giacomin, Landau damping in the Kuramoto model, Ann. Henri Poincaré 17 (2016), no. 7, 1793-1823, DOI 10.1007/s00023-015-04509. MR3510470

[49] R. Fjørtoft, Application of integral theorems in deriving criteria of stability for laminar flows and for the baroclinic circular vortex, Geofys. Publ. Norske Vid.-Akad. Oslo 17 (1950), no. 6, 52. MR0053717

[50] S. Friedlander, N. Pavlović, and R. Shvydkoy, Nonlinear instability for the Navier-Stokes equations, Comm. Math. Phys. 264 (2006), no. 2, 335-347, DOI 10.1007/s00220-006-1526-7. MR.2215608

[51] S. Friedlander, W. Strauss, and M. Vishik, Nonlinear instability in an ideal fluid (English, with English and French summaries), Ann. Inst. H. Poincaré Anal. Non Linéaire 14 (1997), no. 2, 187-209, DOI 10.1016/S0294-1449(97)80144-8. MR.1441392

[52] S. Friedlander and M. M. Vishik, Instability criteria for the flow of an inviscid incompressible fluid, Phys. Rev. Lett. 66 (1991), no. 17, 2204-2206, DOI 10.1103/PhysRevLett.66.2204. MR:1102381

[53] I. Gallagher, T. Gallay, and F. Nier, Spectral asymptotics for large skew-symmetric perturbations of the harmonic oscillator, Int. Math. Res. Not. IMRN 12 (2009), 2147-2199, DOI 10.1093/imrn/rnp013. MR 2511908

[54] T. Gallay, Enhanced dissipation and axisymmetrization of two-dimensional viscous vortices, Arch. Ration. Mech. Anal. 230 (2018), no. 3, 939-975, DOI 10.1007/s00205-018-1262-0. MR.3851053

[55] T. Gebhardt and S. Grossmann, Chaos transition despite linear stability, Phys. Rev. E, 50 (1994), no. 5, 3705 .

[56] A. D. Gilbert, Spiral structures and spectra in two-dimensional turbulence, J. Fluid Mech. 193 (1988), 475-497, DOI 10.1017/S0022112088002228. MR.985193

[57] R. Glassey and J. Schaeffer, Time decay for solutions to the linearized Vlasov equation, Transport Theory Statist. Phys. 23 (1994), no. 4, 411-453, DOI 10.1080/00411459408203873. MR 1264846

[58] R. Glassey and J. Schaeffer, On time decay rates in Landau damping, Comm. Partial Differential Equations 20 (1995), no. 3-4, 647-676, DOI 10.1080/03605309508821107. MR.1318084 
[59] F. Golse, P.-L. Lions, B. Perthame, and R. Sentis, Regularity of the moments of the solution of a transport equation, J. Funct. Anal. 76 (1988), no. 1, 110-125, DOI 10.1016/00221236(88)90051-1. MR.923047

[60] F. Golse, B. Perthame, and R. Sentis, Un résultat de compacité pour les équations de transport et application au calcul de la limite de la valeur propre principale d'un opérateur de transport (French, with English summary), C. R. Acad. Sci. Paris Sér. I Math. 301 (1985), no. 7, 341-344. MR808622

[61] E. Grenier, On the nonlinear instability of Euler and Prandtl equations, Comm. Pure Appl. Math. 53 (2000), no. 9, 1067-1091, DOI 10.1002/1097-0312(200009)53:9〈1067::AIDCPA1)3.3.CO;2-H. MR 1761409

[62] E. Grenier, Y. Guo, and T. T. Nguyen, Spectral instability of general symmetric shear flows in a two-dimensional channel, Adv. Math. 292 (2016), 52-110, DOI 10.1016/j.aim.2016.01.007. MR 3464020

[63] Y. Guo, Variational method for stable polytropic galaxies, Arch. Ration. Mech. Anal. 150 (1999), no. 3, 209-224, DOI 10.1007/s002050050187. MR1738118

[64] Y. Guo and G. Rein, Isotropic steady states in galactic dynamics, Comm. Math. Phys. 219 (2001), no. 3, 607-629, DOI 10.1007/s002200100434. MR1838751

[65] W. Heisenberg, On the stability of laminar flow, in Scientific Review Papers, Talks, and Books, Wissenschaftliche Übersichtsartikel, Vorträge und Bücher, pp. 471-475. Springer, 1984 .

[66] D. D. Holm, J. E. Marsden, T. Ratiu, and A. Weinstein, Nonlinear stability of fluid and plasma equilibria, Phys. Rep. 123 (1985), no. 1-2, 116, DOI 10.1016/0370-1573(85)90028-6. MR794110

[67] H. J. Hwang and J. J. L. Velázquez, On the existence of exponentially decreasing solutions of the nonlinear Landau damping problem, Indiana Univ. Math. J. 58 (2009), no. 6, 2623-2660, DOI 10.1512/iumj.2009.58.3835. MR2603762

[68] S. Ibrahim, Y. Maekawa, and N. Masmoudi, On pseudospectral bound for nonself-adjoint operators and its application to stability of kolmogorov flows, arXiv:1710.05132 (2017).

[69] A. Ionescu and H. Jia, Inviscid damping near shear flows in a channel, arXiv:1808.04026 (2018).

[70] Lord Kelvin, Stability of fluid motion-rectilinear motion of viscous fluid between two parallel plates, Phil. Mag. 24 (1887), 188.

[71] A. Kiselev and V. Šverák, Small scale creation for solutions of the incompressible twodimensional Euler equation, Ann. of Math. (2) 180 (2014), no. 3, 1205-1220, DOI 10.4007/annals.2014.180.3.9. MR3245016

[72] P. Klebanoff, K. Tidstrom, and L. Sargent, The three-dimensional nature of boundary-layer instability, Journal of Fluid Mechanics, 12 (1962), no. 1, 1-34.

[73] G. Kreiss, A. Lundbladh, and D. S. Henningson, Bounds for the threshold amplitudes in subcritical shear flows, J. Fluid Mech. 270 (1994), 175-198, DOI 10.1017/S0022112094004234. MR. 1287784

[74] M. T. Landahl, A note on an algebraic instability of inviscid parallel shear flows, J. Fluid Mech. 98 (1980), no. 2, 243-251, DOI 10.1017/S0022112080000122. MR576172

[75] L. Landau, On the vibrations of the electronic plasma, Acad. Sci. USSR. J. Phys. 10 (1946), 25-34. MR.0023765

[76] M. Latini and A. Bernoff, Transient anomalous diffusion in Poiseuille flow, Journal of Fluid Mechanics, 441 (2001), 399-411.

[77] T. Li, D. Wei, and Z. Zhang, Pseudospectral and spectral bounds for the oseen vortices operator, arXiv:1701.06269 (2017).

[78] T. Li, D. Wei, and Z. Zhang, Pseudospectral bound and transition threshold for the $3 d$ Kolmogorov flow, arXiv:1801.05645 (2018).

[79] M. Liefvendahl and G. Kreiss, Bounds for the threshold amplitude for plane Couette flow, J. Nonlinear Math. Phys. 9 (2002), no. 3, 311-324, DOI 10.2991/jnmp.2002.9.3.5. MR1916388

[80] C. C. Lin, On the stability of two-dimensional parallel flows, Proc. Nat. Acad. Sci. U. S. A. 30 (1944), 316-323, DOI 10.1073/pnas.30.10.316. MR.0011614

[81] Z. Lin and M. Xu, Metastability of Kolmogorov flows and inviscid damping of shear flows, arXiv:1707.00278 (2017).

[82] Z. Lin and C. Zeng, Inviscid dynamical structures near Couette flow, Arch. Ration. Mech. Anal. 200 (2011), no. 3, 1075-1097, DOI 10.1007/s00205-010-0384-9. MR2796139 
[83] R. Lindzen, Instability of plane parallel shear flow (toward a mechanistic picture of how it works), PAGEOPH 126 (1988), 1.

[84] A. Lundbladh, D. S. Henningson, and S. C. Reddy, Threshold amplitudes for transition in channel flows, in Transition, turbulence and combustion, pp. 309-318. Springer, 1994.

[85] T. Lundgren, Strained spiral vortex model for turbulent fine structure, Phys. of Fl. 25 (1982), 2193.

[86] D. Lynden-Bell, Statistical mechanics of violent relaxation in stellar systems, Mon. Not. R. Astr. Soc. 136 (1967), 101-121.

[87] A. J. Majda and A. L. Bertozzi, Vorticity and incompressible flow, Cambridge Texts in Applied Mathematics, vol. 27, Cambridge University Press, Cambridge, 2002. MR 1867882

[88] J. Malmberg, C. Wharton, C. Gould, and T. O'Neil, Plasma wave echo, Phys. Rev. Lett. 20 (1968), no. 3, 95-97.

[89] C. Marchioro and M. Pulvirenti, Mathematical theory of incompressible nonviscous fluids, Applied Mathematical Sciences, vol. 96, Springer-Verlag, New York, 1994. MR.1245492

[90] J. D. McCalpin, On the adjustment of azimuthally perturbed vortices, Journal of Geophysical Research: Oceans, 92 (1987), C8, 8213-8225.

[91] M. Melander, J. McWilliams, and N. Zabusky, Axisymmetrization and vorticity-gradient intensification of an isolated two-dimensional vortex through filamentation, Journal of Fluid Mechanics 178 (1987), 137-159.

[92] P. J. Morrison, Hamiltonian description of the ideal fluid, Rev. Modern Phys. 70 (1998), no. 2, 467-521, DOI 10.1103/RevModPhys.70.467. MR1627532

[93] C. Mouhot and C. Villani, On Landau damping, Acta Math. 207 (2011), no. 1, 29-201, DOI 10.1007/s11511-011-0068-9. MR2863910

[94] N. S. Nadirashvili, Wandering solutions of the two-dimensional Euler equation (Russian), Funktsional. Anal. i Prilozhen. 25 (1991), no. 3, 70-71, DOI 10.1007/BF01085491; English transl., Funct. Anal. Appl. 25 (1991), no. 3, 220-221 (1992). MR1139875

[95] W. Orr, The stability or instability of steady motions of a perfect liquid and of a viscous liquid, Part I: a perfect liquid, Proc. Royal Irish Acad. Sec. A: Math. Phys. Sci. 27 (1907), $9-68$.

[96] S. A. Orszag and L. C. Kells, Transition to turbulence in plane Poiseuille and plane Couette flow, Journal of Fluid Mechanics, 96 (1980), no. 1, 159-205.

[97] O. Penrose, Electrostatic instability of a uniform nonMaxwellian plasma, Phys. Fluids 3 (1960), 258-265.

[98] D. S. Pradeep and F. Hussain, Transient growth of perturbations in a vortex column, J. Fluid Mech. 550 (2006), 251-288, DOI 10.1017/S0022112005008207. MR2263985

[99] L. Rayleigh, On the Stability, or Instability, of certain Fluid Motions, Proc. Lond. Math. Soc. 11 (1879/80), 57-70, DOI 10.1112/plms/s1-11.1.57. MR.1575266

[100] L. Rayleigh. On convection currents in a horizontal layer of fluid, when the higher temperature is on the under side, The London, Edinburgh, and Dublin Philosophical Magazine and Journal of Science, 32 (1916), no. 192, 529-546.

[101] S. C. Reddy, P. J. Schmid, J. S. Baggett, and D. S. Henningson, On stability of streamwise streaks and transition thresholds in plane channel flows, J. Fluid Mech. 365 (1998), 269-303, DOI 10.1017/S0022112098001323. MR1631950

[102] O. Reynolds, An experimental investigation of the circumstances which determine whether the motion of water shall be direct or sinuous, and of the law of resistance in parallel channels, Proc. R. Soc. Lond. 174 (1883), 935-982.

[103] P. Rhines and W. Young, How rapidly is a passive scalar mixed within closed streamlines?, Journal of Fluid Mechanics 133 (1983), 133-145.

[104] V. A. Romanov, Stability of plane-parallel Couette flow (Russian), Funkcional. Anal. i Priložen. 7 (1973), no. 2, 62-73. MR0326191

[105] D. Ryutov, Landau damping: half a century with the great discovery, Plasma Physics and Controlled Fusion 41 (1999), no. 3A.

[106] D. A. Schecter, D. H. E. Dubin, A. C. Cass, C. F. Driscoll, I. M. Lansky, and T. M. O'Neil, Inviscid damping of asymmetries on a two-dimensional vortex, Phys. Fluids 12 (2000), no. 10, 2397-2412, DOI 10.1063/1.1289505. MR.1789996

[107] P. J. Schmid and D. S. Henningson, Stability and transition in shear flows, Applied Mathematical Sciences, vol. 142, Springer-Verlag, New York, 2001. MR.1801992 
[108] R. Shvydkoy and S. Friedlander, Problem for the linearized Euler equation, Nonlinear Partial Differential Equations and Related Analysis: The Emphasis Year 2002-2003 Program on Nonlinear Partial Differential Equations and Related Analysis, September 2002-July 2003, Northwestern University, Evanston, Illinois, vol. 371, p. 271, 2005.

[109] G. B. Smith and M. T. Montgomery, Vortex axisymmetrization: Dependence on azimuthal wave-number or asymmetric radial structure changes, Quarterly Journal of the Royal Meteorological Society 121 (1995), no. 527, 1615-1650.

[110] H. Squire, On the stability for three-dimensional disturbances of viscous fluid flow between parallel walls, Proceedings of the Royal Society of London. Series A, Containing Papers of a Mathematical and Physical Character 142 (1933), no. 847, 621-628.

[111] T. Tao, Nonlinear dispersive equations, CBMS Regional Conference Series in Mathematics, vol. 106, Published for the Conference Board of the Mathematical Sciences, Washington, DC; by the American Mathematical Society, Providence, RI, 2006. Local and global analysis. MR2233925

[112] N. Tillmark and P. Alfredsson, Experiments on transition in plane Couette flow, J. Fluid Mech. 235 (1992), 89-102.

[113] L. N. Trefethen and M. Embree, Spectra and pseudospectra: The behavior of nonnormal matrices and operators, Princeton University Press, Princeton, NJ, 2005. MR2155029

[114] L. N. Trefethen, A. E. Trefethen, S. C. Reddy, and T. A. Driscoll, Hydrodynamic stability without eigenvalues, Science 261 (1993), no. 5121, 578-584, DOI 10.1126/science.261.5121.578. MR.1229495

[115] N. G. van Kampen, On the theory of stationary waves in plasmas, Physica 21 (1955), 949-963. MR0075080

[116] J. Vanneste, Nonlinear dynamics of anisotropic disturbances in plane Couette flow, SIAM J. Appl. Math. 62 (2001/02), no. 3, 924-944, DOI 10.1137/S0036139900381420. MR1897729

[117] J. Vanneste, P. Morrison, and T. Warn, Strong echo effect and nonlinear transient growth in shear flows, Physics of Fluids, 10 (1998), no. 6, 1398.

[118] J. Vukadinovic, E. Dedits, A. C. Poje, and T. Schäfer, Averaging and spectral properties for the 2D advection-diffusion equation in the semi-classical limit for vanishing diffusivity, Phys. D 310 (2015), 1-18, DOI 10.1016/j.physd.2015.07.011. MR3396847

[119] F. Waleffe, Transition in shear flows. Nonlinear normality versus nonnormal linearity, Phys. Fluids 7 (1995), no. 12, 3060-3066, DOI 10.1063/1.868682. MR.1361366

[120] Y. H. Wan and M. Pulvirenti, Nonlinear stability of circular vortex patches, Comm. Math. Phys. 99 (1985), no. 3, 435-450. MR795112

[121] Y. Wang, Rapid filamentation zone in a numerically simulated tropical cyclone, Journal of the Atmospheric Sciences 65 (2008), no. 4, 1158-1181.

[122] D. Wei and Z. Zhang, Transition threshold for the 3D Couette flow in Sobolev space, arXiv:1803.01359 (2018).

[123] D. Wei, Z. Zhang, and W. Zhao, Linear inviscid damping for a class of monotone shear flow in Sobolev spaces, Comm. Pure Appl. Math. 71 (2018), no. 4, 617-687, DOI 10.1002/cpa.21672. MR3772399

[124] D. Wei, Z. Zhang, and W. Zhao, Linear inviscid damping and enhanced dissipation for the Kolmogorov flow, arXiv:1711.01822 (2017).

[125] D. Wei, Z. Zhang, and W. Zhao, Linear inviscid damping and vorticity depletion for shear flows, arXiv:1704.00428 (2017).

[126] A. M. Yaglom, Hydrodynamic instability and transition to turbulence, Fluid Mechanics and its Applications, vol. 100, Springer, Dordrecht, 2012. With a foreword by Uriel Frisch and a memorial note for Yaglom by Peter Bradshaw. MR3185102

[127] J. Yang and Z. Lin, Linear inviscid damping for Couette flow in stratified fluid, J. Math. Fluid Mech. 20 (2018), no. 2, 445-472, DOI 10.1007/s00021-017-0328-3. MR3808578

[128] H. Yao and N. Zabusky, Axisymmetrization of an isolated vortex region by splitting and partial merging of satellite depletion perturbations, Physics of Fluids 8 (1996), no. 7, 18421847.

[129] B. Young, Landau damping in relativistic plasmas, J. Math. Phys. 57 (2016), no. 2, 021502 , 68, DOI 10.1063/1.4939275. MR3448678

[130] J. Yu and C. Driscoll, Diocotron wave echoes in a pure electron plasma, IEEE Trans. Plasma Sci. 30 (2005), no. 1, 24-25. 
[131] J. Yu, C. Driscoll, and T. O‘Neil, Phase mixing and echoes in a pure electron plasma, Phys. of Plasmas 12 (2005), 055701.

[132] C. Zillinger, Linear inviscid damping for monotone shear flows, Trans. Amer. Math. Soc. 369 (2017), no. 12, 8799-8855, DOI 10.1090/tran/6942. MR3710645

[133] C. Zillinger, Linear inviscid damping for monotone shear flows in a finite periodic channel, boundary effects, blow-up and critical Sobolev regularity, Arch. Ration. Mech. Anal. 221 (2016), no. 3, 1449-1509, DOI 10.1007/s00205-016-0991-1. MR.3509006

[134] C. Zillinger, Linear inviscid damping for monotone shear flows in a finite periodic channel, boundary effects, blow-up and critical Sobolev regularity, Arch. Ration. Mech. Anal. 221 (2016), no. 3, 1449-1509, DOI 10.1007/s00205-016-0991-1. MR3509006

[135] C. Zillinger, On circular flows: linear stability and damping, J. Differential Equations 263 (2017), no. 11, 7856-7899, DOI 10.1016/j.jde.2017.08.026. MR.3705700

[136] C. Zillinger, Linear inviscid damping for monotone shear flows, Trans. Amer. Math. Soc. 369 (2017), no. 12, 8799-8855, DOI 10.1090/tran/6942. MR3710645

[137] A. Zlatoš, Diffusion in fluid flow: dissipation enhancement by flows in 2D, Comm. Partial Differential Equations 35 (2010), no. 3, 496-534, DOI 10.1080/03605300903362546. $\operatorname{MR} 2748635$

4176 Campus Drive - William E. Kirwan Hall, College Park, Maryland 20742

Email address: jacob@cscamm.umd.edu

Courant Institute of Mathematical Sciences, 251 Mercer Street, New York, New YORK 10012

Email address: pgermain@cims.nyu.edu

Department of mathematics, New York University in Abu Dhabi, SaAdyiat Island, Abu Dhabi, United Arab Emirates

Email address: masmoudi@cims.nyu.edu 